\title{
Tracing HIV-1 strains that imprint broadly neutralizing antibody responses
}

Roger D. Kouyos ${ }^{1,2,15,16 *}$, Peter Rusert ${ }^{1,15}$, Claus Kadelka ${ }^{1,2,15}$, Michael Huber ${ }^{1}$, Alex Marzel ${ }^{1,2}$, Hanna Ebner ${ }^{1}$, Merle Schanz ${ }^{1}$, Thomas Liechti ${ }^{1,13}$, Nikolas Friedrich ${ }^{1}$, Dominique L. Braun ${ }^{1,2}$, Alexandra U. Scherrer ${ }^{1,2}$, Jacqueline Weber ${ }^{1}$, Therese Uhr ${ }^{1}$, Nicolas S. Baumann ${ }^{1}$, Christine Leemann ${ }^{1,2}$, Herbert Kuster ${ }^{1,2}$, Jean-Philippe Chave ${ }^{3}$, Matthias Cavassini ${ }^{4}$, Enos Bernasconi ${ }^{5}$, Matthias Hoffmann ${ }^{6}$, Alexandra Calmy ${ }^{7}$, Manuel Battegay ${ }^{8}$, Andri Rauch ${ }^{9}$, Sabine Yerly ${ }^{10}$, Vincent Aubert ${ }^{11}$, Thomas Klimkait ${ }^{12}$, Jürg Böni ${ }^{1}$, Karin J. Metzner ${ }^{1,2}$, Huldrych F. Günthard ${ }^{1,2,16 *}$, Alexandra Trkola ${ }^{1,16 *}$ \& The Swiss HIV Cohort Study ${ }^{14}$

Understanding the determinants of broadly neutralizing antibody (bNAb) evolution is crucial for the development of bNAb-based HIV vaccines ${ }^{1}$. Despite emerging information on cofactors that promote bNAb evolution in natural HIV-1 infections, in which the induction of bNAbs is genuinely rare $^{2}$, information on the impact of the infecting virus strain on determining the breadth and specificity of the antibody responses to HIV-1 is lacking. Here we analyse the influence of viral antigens in shaping antibody responses in humans. We call the ability of a virus strain to induce similar antibody responses across different hosts its antibody-imprinting capacity, which from an evolutionary biology perspective corresponds to the viral heritability of the antibody responses. Analysis of 53 measured parameters of HIV-1-binding and neutralizing antibody responses in a cohort of $303 \mathrm{HIV}-1$ transmission pairs (individuals who harboured highly related HIV-1 strains and were putative direct transmission partners or members of an HIV-1 transmission chain) revealed that the effect of the infecting virus on the outcome of the bNAb response is moderate in magnitude but highly significant. We introduce the concept of bNAb-imprinting viruses and provide evidence for the existence of such viruses in a systematic screening of our cohort. The bNAb-imprinting capacity can be substantial, as indicated by a transmission pair with highly similar HIV-1 antibody responses and strong $b N A b$ activity. Identification of viruses that have bNAb-imprinting capacities and their characterization may thus provide the potential to develop lead immunogens.

The capacity to evoke highly similar bNAb responses across vaccinees is crucial for an effective HIV-1 immunogen. Closely related HIV-1 strains may induce similar neutralization responses, as observations from mother-to-child transmission suggest ${ }^{3}$. To formally evaluate the virus-dictated heritability of antibody responses, we investigated the imprinting capacity of HIV-1 antibody responses within transmission pairs. We designed our study to address two central problems (Extended Data Fig. 1a, b). First, we investigated whether the same virus, when transmitted to two different people, induces similar binding and neutralizing antibody responses (imprints a similar antibody response). Second, we investigated how promising HIV-1 strains with superior bNAb-imprinting capacity can be identified.

On the basis of the Swiss $4.5 \mathrm{~K} \mathrm{Screen}^{4,5}$, we established a large, adult transmission-pair cohort $(n=303$ putative transmission pairs) with comprehensive information on HIV-1-binding and neutralizing antibody responses (Extended Data Fig. 1a). Extensive data on HIV-binding antibody reactivity encompassing IgG1, IgG2 and IgG3 reactivity with 13 antigens was available for all 606 patients from previous analyses ${ }^{5}$ (Supplementary Data 1 ). Neutralization activity was assessed against a 14 multi-clade virus panel (Extended Data Fig. 2a and Supplementary Data 1,2) and evaluated by breadth and a cumulative neutralization score, reflecting potency and breadth across the analysed virus panel (Extended Data Fig. 2a-d). Overall, the neutralization activity in the transmission pair cohort showed the typical pattern seen in chronic infection ${ }^{4}$ : the majority of patients displayed no or low neutralization activity ( $73 \%$ of patients had below $10 \%$ breadth).

We hypothesized that if virus-associated factors are important in determining antibody responses, HIV antibody response patterns should be similar in transmission pairs. Using the established 53 HIV-1 antibody parameters (14 neutralization and 39 binding antibody parameters), we conducted a systematic assessment of the HIV-1 antibody imprinting capacity in transmission pairs (Extended Data Fig. 2e).

We detected a significant, positive association of the transmitter and recipient neutralization responses to 7 of the 14 panel viruses (Fig. 1a and Extended Data Fig. 3a). The overall similarity of the neutralization fingerprint within pairs across the 14 panel viruses was assessed as average Spearman correlation $\left(\rho_{\text {Spearman-average }}\right)$ of their neutralization activity (Fig. 1b). To determine the statistical significance of the observed similarity, we used shuffling approaches that randomly reassign recipients to transmitters, thus generating a distribution for the null-expectation of no association. Neutralization fingerprints in observed transmission pairs proved on average positively and significantly associated $\left(\rho_{\text {Spearman-average }}=0.11, P_{\text {shuffling }}<0.001\right.$; Fig. 1 b). To confirm the influence of the infecting virus, we estimated the heritability of antibody responses by two alternative methods adjusting for the influence of various host, viral and disease factors that are known to influence antibody responses ${ }^{4,5}$. First, we restricted the shuffling to pairs with the same infection length, subtype and ethnicity (Fig. 1b). Second, we considered mixed-effect Tobit models adjusted for key drivers of HIV-1 antibody development (infection length, ethnicity, virus load and viral diversity) and bNAb specificity (HIV-1 pol subtype) (Fig. 1c). Both approaches confirmed a significant, within-pair correlation of neutralization (Fig. 1b, c). Although other, not yet defined, non-virus-associated factors common to both transmission partners may exist, our data strongly suggest that the infecting virus strain affects the development of neutralization responses. The effects (Fig. 1a, b) remained robust when restricting the analysis to pairs infected with subtype B virus, indicating that the effect is not driven by specific subtypes (Extended Data Fig. 4a, b).

\footnotetext{
${ }^{1}$ Institute of Medical Virology, University of Zurich, Zurich, Switzerland. ${ }^{2}$ Division of Infectious Diseases and Hospital Epidemiology, University Hospital Zurich, University of Zurich, Zurich, Switzerland. ${ }^{3}$ Clinique de La Source, Lausanne, Switzerland. ${ }^{4}$ Division of Infectious Diseases, University Hospital Lausanne, University of Lausanne, Lausanne, Switzerland. ${ }^{5}$ Division of Infectious Diseases, Regional Hospital Lugano, Lugano, Switzerland. ${ }^{6}$ Division of Infectious Diseases, Cantonal Hospital St. Gallen, St. Gallen, Switzerland. ${ }^{7}$ Division of Infectious Diseases, University Hospital

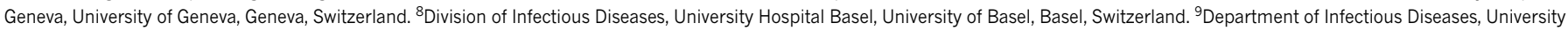
Hospital Bern, University of Bern, Bern, Switzerland. ${ }^{10}$ Laboratory of Virology, Division of Infectious Diseases, University Hospital Geneva, University of Geneva, Geneva, Switzerland. ${ }^{11}$ Division of Immunology and Allergy, University Hospital Lausanne, University of Lausanne, Lausanne, Switzerland. ${ }^{12}$ Division of Infection Diagnostics, Department of Biomedicine-Petersplatz, University of Basel, Basel, Switzerland. ${ }^{13}$ Present address: ImmunoTechnology Section, Vaccine Research Center, NIAID, National Institutes of Health, Bethesda, MD, USA. ${ }^{14} \mathrm{~A}$ Iist of participants and their affiliations appears at the end of the paper. ${ }^{15}$ These authors contributed equally: Roger D. Kouyos, Peter Rusert, Claus Kadelka. ${ }^{16}$ These authors jointly supervised this work: Roger D. Kouyos, Huldrych F. Günthard, Alexandra Trkola. *email:roger.kouyos@usz.ch; huldrych.guenthard@usz.ch; trkola.alexandra@virology.uzh.ch
} 
a

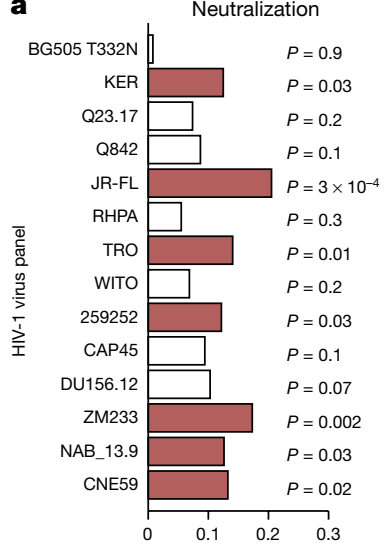
individual virus strains in transmitters and recipients
Correlation $\left(\rho_{\text {Spearman }}\right)$ of neutralization response to
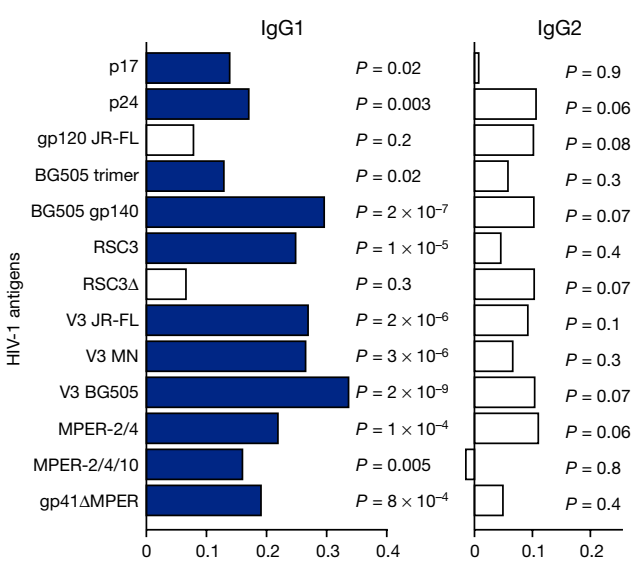

Correlation ( $\rho_{\text {Spearman }}$ ) of binding response to individual antigens in transmitters and recipients

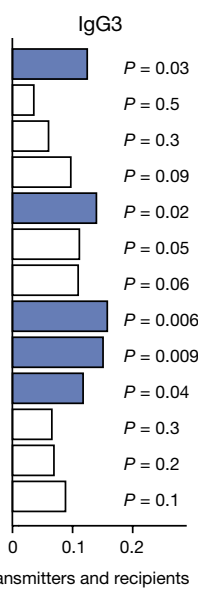

$P=0.03$

$P=0.5$

$=0.3$

$=0.05$

$=0.06$

$=0.009$

$=0.3$

0.2

0.1
0.006

b

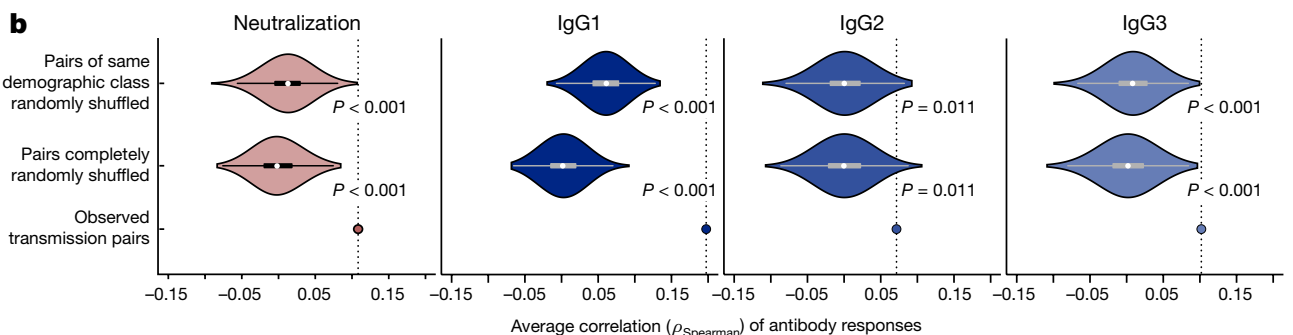

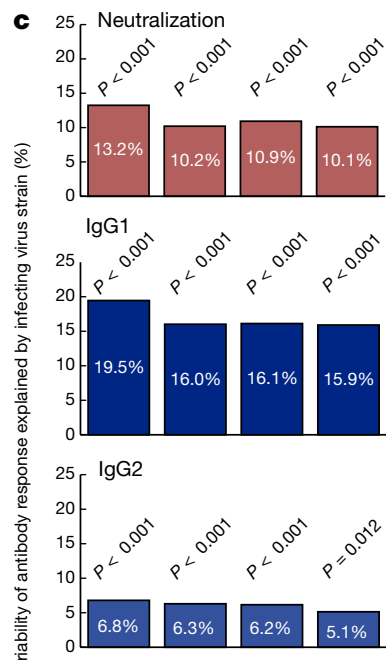

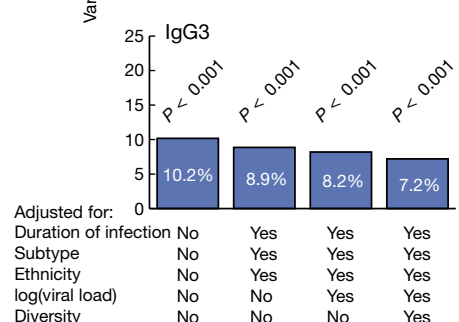

Fig. 1 | Similarity of neutralization and antibody-binding responses in transmission pairs. a, Spearman correlation of antibody responses in observed transmission pairs ( $n=303$; see also Extended Data Figs. 2e, 3a). Significant correlations (two-sided $\left.P_{\text {Spearman }}<0.05\right)$ are coloured. b, Average Spearman correlation of antibody responses in observed transmission pairs $(n=303)$ compared to two alternative scenarios: (1) completely random reassignment of recipients to transmitters and (2) random reassignment of recipients to transmitters with the same demographics (subtype, ethnicity, and untreated infection length). Violins (smoothed using a normal kernel) and one-sided $P$ values were derived from 1,000 random reassignments of recipients to transmitters. Medians (white dots) and boxes spanning the interquartile range (IQR) range are shown. Each whisker extends to the most extreme value no more than $1.5 \times$ IQR from the box. c, Proportion of variability in antibody responses explained by the infecting virus, determined using unadjusted and differently adjusted mixed-effect Tobit regression models $(n=303)$. One-sided $P$ values were derived from comparison with 1,000 random reassignments of recipients to transmitters.
Unravelling the quantitative contribution of the infecting virus to the neutralization response is of particular importance for vaccine development. The mixed-effect Tobit models revealed that, on average, 13.2\% of the variability of the neutralization response can be explained by the infecting virus (Fig. 1c). Notably, various alternative models adjusted for cofactors of HIV-1 antibody induction-such as viral load or duration of infection-yielded similar results (9.3-13.8\% neutralization variability explained by the virus; Extended Data Fig. 3b). Comparable results were also obtained when we restricted the analysis to the 184 pairs in which both individuals were infected for three or more years (neutralization heritability in Tobit models: 13.2\% unadjusted, 9.3\% fully adjusted) or when we used multiple imputation instead of a complete case analysis in the fully adjusted model (10.9\% neutralization variability; Extended Data Fig. 3c).

HIV-1 antigen-binding activities were also significantly correlated within transmission pairs, but the degree of correlation differed considerably across antigens and IgG classes (Fig. 1a). IgG1 reactivity, the most prominent IgG response in HIV-1 infection ${ }^{5}$, displayed the highest similarity in transmission pairs (Fig. 1a, b), followed by IgG3 and IgG2 responses. Of note, IgG2 responses, which are only present at low levels during HIV-1 infection, showed no statistically significant similarity at the level of individual antigens. The average within-pair similarity across antigens was nevertheless significantly positive for all three $\operatorname{IgG}$ subclasses, with and without taking potentially confounding parameters into account (Fig. 1b). As observed for neutralization responses, the findings remained significant when the analysis was restricted to subtype B infection (Extended Data Fig. 4c, d). The effect of the infecting virus was comparable in magnitude to the effect on neutralization (19\%, 7\% and $10 \%$ for IgG1, IG2 and IgG3 responses, respectively), with similar values observed after adjustment for analysed cofactors of HIV-1 antibody induction (Fig. 1c). The identified influence of the infecting virus on the heritability of HIV-1 binding and neutralizing antibody responses was robust, as confirmed by additional sensitivity analyses (Extended Data Figs. 3b, 5a-c).

The overall effect sizes of the influence of virus genetics on neutralizing and binding antibodies that we report here must be considered as lower bound estimates, as neutralization breadth is generally low in HIV-1 infection. Furthermore, as our cohort setup did not allow assessing matched time points, transmission partners had experienced different lengths of virus replication and antibody response development at sample collection. Although the effects that we identified across the cohort were moderate, individual cases may substantially exceed the observed average antibody similarity. In support of this, two HIV-1 subtype B-infected elite neutralizers (top 1\% of neutralizers identified in the Swiss $4.5 \mathrm{~K} \mathrm{Screen}{ }^{4}$ ) formed one transmission pair ( $0.0058 \mathrm{HIV}-1$ pol genetic distance) that stood out in both potency and breadth (Fig. 2a, b). This pair was subsequently verified by their shared treating physician as a mixed ethnicity heterosexual couple in a stable partnership (T282 female (North African) to R282 male (Asian) transmission). Identification of transmission pairs and genetic subtype information in our cohort is based on pol sequence data collected early after diagnosis. We performed full-genome next-generation sequencing of plasma viruses from T282 and R282 at the time point analysed for antibody reactivity to rule out later superinfection. The obtained consensus full-genome sequences confirmed closely related subtype B viruses in T282 and R282 (Extended Data Fig. 6a). Because the analysed samples of T282 and R282 were collected 1.9 and 3.4 years after the putative transmission event, respectively, considerable genetic differences in Env between the pair were expected ${ }^{6}$. Env similarity in the pair was nevertheless evident, as highlighted by phylogenetic comparison, 
a

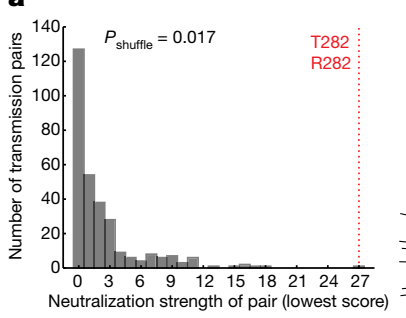

b

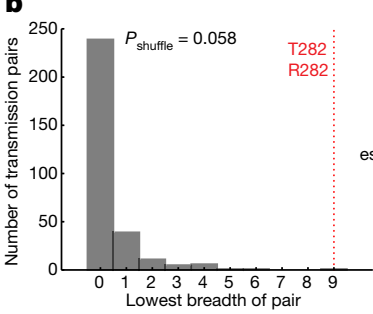

c

T282 1.9 years post

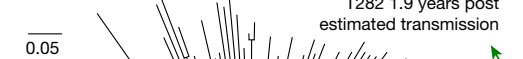

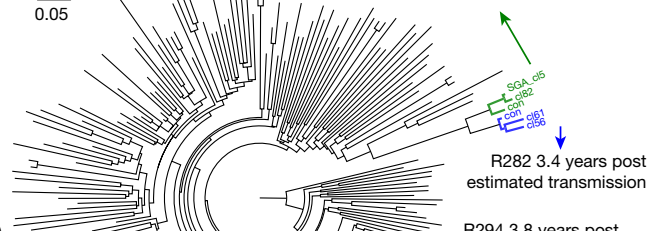

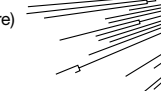
R294 3.8 years post estimated transmission

d

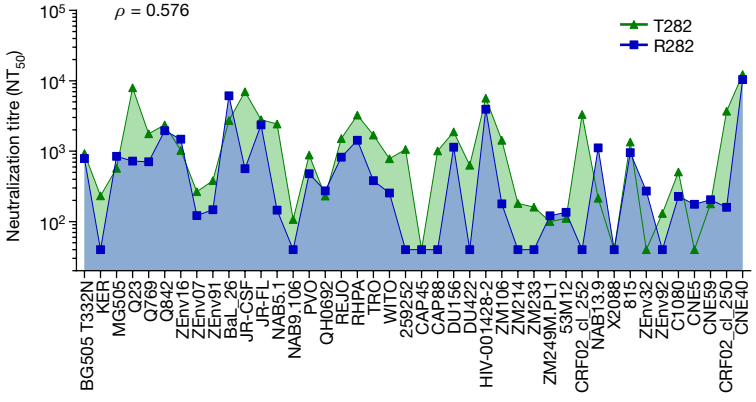

R11 8.1 years post timated transmission

T117.9 years post stimated transmission

Fig. 2 Identification of an elite-neutralizing transmission pair, T282 and R282. a, b, Lowest neutralization strength (a) and lowest breadth (b) of recipients and transmitters in observed transmission pairs $(n=303$; red line: elite-neutralizing pair T282 and R282). One-sided $P$ values were derived from comparison of maximum value with $10^{6}$ random reassignments of recipients to transmitters. c, Phylogenetic analysis of Env sequences from pairs T282 and R282 (green and blue), T11 and R11

validating the close relatedness of the viruses infecting the transmission partners (Fig. 2c). The same pattern was observed for two other transmission pairs (T11-R11 and T294-R294) analysed for comparison.

In line with reactivity to an originally highly related Env antigen, neutralization fingerprint analysis of plasma samples from T282 and R282 in a 42-virus panel confirmed highly similar neutralization responses in this pair $\left(\rho_{\text {Spearman }}=0.576\right.$; Fig. $\left.2 \mathrm{~d}\right)$. Delineation of plasma bNAb
, and T294 and R294 (yellow), combined with 198 closely related Env background sequences (see Supplementary Methods and Extended Data Fig. 6). Years post estimated transmission at sampling indicated for transmission pairs. d, The $50 \%$ neutralization titres of plasma from T282 and R282 against the multi-clade virus panel $\left(n=42 ; \rho_{\text {Spearman }}=0.576\right)$. e, Neutralization fingerprint similarity $\left(\rho_{\text {Spearman }}\right)$ of plasma from T282 and R282 with 25 known bNAbs.

specificity by fingerprint analyses (Fig. 2e and Supplementary Data 2) and mutant-virus neutralization mapping (Extended Data Fig. 7a) showed that both partners developed a CD4 binding-site-directed bNAb plasma response and that additional V3 glycan bNAb activity was present in the transmitter.

The occurrence of a transmission pair with such pronounced neutralization strength and similarity in neutralization responses is a

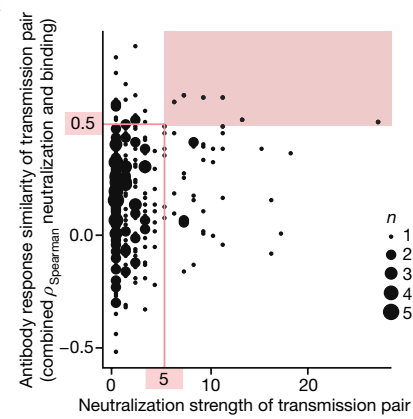
(lowest common score)

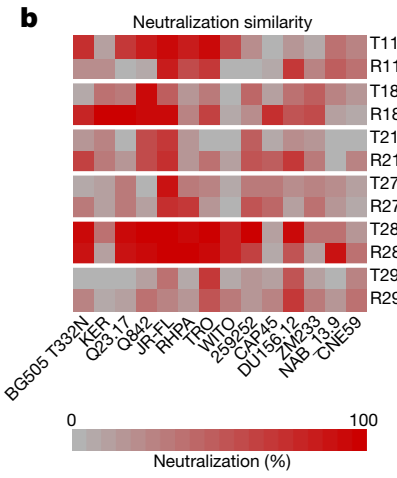

Neutralization $(\%)$
IgG1 binding similarity

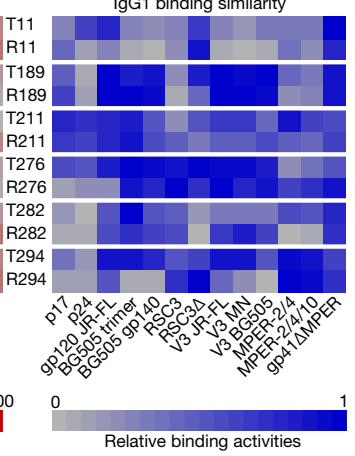

c

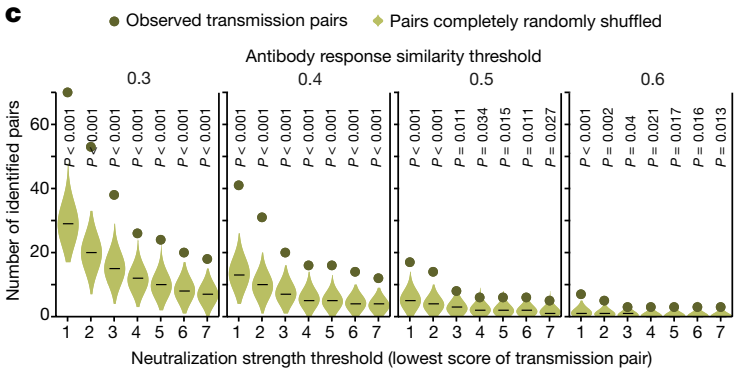

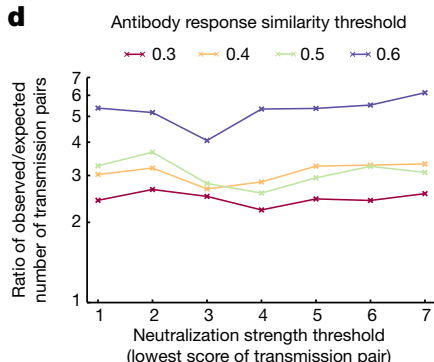

(lowest score of transmission pair)
Fig. 3 | Systematic screen for virus strains with bNAb-imprinting capacity. a, Antibody response similarity versus neutralization strength of 303 transmission pairs (see Extended Data Fig. 8). b, Comparison of neutralization and IgG1-binding profiles for pairs with a similarity index $\geq 0.5$ and a neutralization strength $\geq 5$ (red area in a). c, Observed (dots) versus expected (violins) number of all $n=303$ pairs with bNAbimprinting capacity for various similarity thresholds (different subplots) and neutralization strength thresholds ( $x$ axes). Violins (smoothed using a normal kernel) and one-sided $P$ values were derived from 1,000 random reassignments of recipients to transmitters. Lines depict medians. d, Ratio of observed versus expected number of pairs with bNAbimprinting capacity for various similarity thresholds (colours) and neutralization strength thresholds ( $x$ axis). 
unlikely to be by chance. By randomly reassigning recipients to transmitters $\left(10^{6}\right.$ replicates $)$, we determined that the probability of such a strong shared neutralization strength by chance is $P_{\text {shuffle }}=0.017$ (Fig. 2a). Although additional non-virus-linked factors that positively influence similar bNAb development may exist, our results point to a considerable influence of the transmitted virus in this elite bNAbinducing pair. This strongly suggests the existence of virus envelopes with strong bNAb-imprinting capacity that will need to be identified for use in vaccine development.

We next developed a systematic, statistical approach (Extended Data Fig. 8) to identify pairs in which both partners developed notable neutralization strength (assessed by the lower neutralization score reached by one partner) and had similar neutralization and binding responses (Fig. 3a). Using various thresholds for the neutralization strength $(n=7)$ and the within-pair similarity index $(n=4)$ of the antibody response, we identified for each combination of thresholds the bNAb-imprinting capacity in our cohort. We counted the number of transmission pairs with a certain neutralization strength and a certain similarity index (exemplified for thresholds of neutralization $=5$ and similarity $=0.5$ in Fig. $3 a, b$ ). For all tested threshold criteria, we identified substantially more candidate transmission pairs with bNAb-imprinting capacity than would be expected by chance (Fig. 3c, d). This indicates that the transmitted HIV-1 strains in these pairs are promising candidates for imprinting highly similar neutralization responses.

Even though the viral Env antigen must certainly have a role, it is conceivable that bNAb-imprinting capacity might also, in part, depend on other virus traits, or demographic, host genomic, or transmission route-related factors. We therefore investigated the influence of known factors implicated in HIV-1 antibody development on bNAb-imprinting capacity in our cohort (Extended Data Fig. 7b, c). BNAb-imprinting capacity was detected more frequently among transmission pairs in which both partners had been infected for three or more years before assessment of antibody reactivity (Extended Data Fig. 7c). This is in line with the generally higher frequency of bNAb induction after prolonged exposure to HIV $-1^{4}$. None of the other tested factors was significantly associated with bNAb-imprinting capacity (Extended Data Fig. 7b, c). Owing to the cross-sectional study set-up, we lacked the means to compare antibody responses at matched time points of infection and, therefore, our findings probably represent an underestimation of the frequency of bNAb-imprinters. Although our transmission cohort is one of the largest described to date, it may still require substantially larger cohorts and specifically tailored studies to detect more subtle influences of cofactors on bNAb imprinting.

The formal assessment of virus-induced heritability of the antibody response performed here quantifies the impact of HIV-1 immunogens on inducing qualitatively and quantitatively similar neutralizing and binding antibody responses. The effect of the infecting virus we observed across the assessed large, cross-sectional, natural history cohort of HIV-1 transmission pairs was significant, ranging between 7 and 19\% depending on the antibody response considered (Fig. 1c). This is lower in magnitude than the effect of virus genetics on HIV-1 set point viral load $(29 \%)^{7,8}$ but comparable to the effect of virus genetics on CD4+ T cell decline $(17 \%)^{7,8}$ and the effect of host genetics of HLA and CCR 5 SNPs $(14.5 \%)^{9}$. Notably, owing to the cross-sectional nature of the transmission cohort, sampling time points of pairs were not matched for duration of infection. Adjustment for infection time controlled for this in the estimate of the overall imprinting capacity (Fig. 1c). Although in patients with shorter infection times, bnAb-imprinting capacity may have been missed (Fig. 3), the fact that we observe a significant genetic effect of the virus in a cross-sectional, natural history cohort is a clear indication of the strength of our results.

Using a statistical approach to systematically screen for virus strains with bnAb-imprinting capacity, we show that only a minority of HIV-1 transmissions exhibit transferability of strong neutralization antibody traits, suggesting that only some virus strains or Env variants harbour bnAb-imprinting capacity. bnAb-imprinting capacity may be a genuine feature of certain Env variants or a temporal issue, as epitopes needed to evoke a specific neutralization response may be presented only transiently until escape mutations establish. Depending on when, with respect to viral evolution in the transmitter, the transmission occurred, the recipient may not have been exposed to the relevant epitope. If virus escape limits the heritability of antibody responses, transmission during acute HIV-1 infection (where neutralization activity is still low and hence neutralization escape is scarce) should show a higher similarity of antibody responses in transmitter and recipients. However, we found no evidence for higher frequencies of bnAb-imprinting capacity amongst acute phase transmission cases in our cohort (Extended Data Fig. 7c), suggesting that virus escape is not the main limiting factor and that higher patient numbers would be needed to uncover more subtle effects. In general, the fact that bnAb-imprinting upon transmission occurs strongly suggests that relevant epitopes that allow germline triggering can be preserved over longer time periods, for instance as a result of partial escape that retains bnAb-binding capacity ${ }^{10,11}$.

Although the average effect of virus genetics across the entire cohort was moderate, individual cases with high antibody similarity and capacity to imprint a bNAb immune response can exist. The eliteneutralizing transmission pair that we identified highlights that distinct strains may exist, which harbour the potential to evoke highly similar bNAb and binding antibody responses across individuals. Further studies that identify more such pairs and a detailed characterization of the transmitted strains, longitudinal envelope evolution and the evoked bNAb responses will be needed to understand which specific features render a strain a bNAb imprinter. Clearly, if bNAb-imprinting strains exist, they need to be specifically investigated and characterized. In particular, envelope proteins from bNAb imprinters with proven in vivo transferability of antibody reactivity may provide the ultimate candidate immunogen(s) on which to base bNAb vaccine design.

\section{Online content}

Any methods, additional references, Nature Research reporting summaries, source data, statements of data availability and associated accession codes are available at https://doi.org/10.1038/s41586-018-0517-0.

Received: 12 February 2018; Accepted: 9 August 2018; Published online 10 September 2018.

1. Burton, D. R. \& Hangartner, L. Broadly neutralizing antibodies to HIV and their role in vaccine design. Annu. Rev. Immunol. 34, 635-659 (2016).

2. Subbaraman, H., Schanz, M. \& Trkola, A. Broadly neutralizing antibodies: what is needed to move from a rare event in HIV-1 infection to vaccine efficacy? Retrovirology 15, 52 (2018).

3. Mabuka, J., Goo, L., Omenda, M. M., Nduati, R. \& Overbaugh, J. HIV-1 maternal and infant variants show similar sensitivity to broadly neutralizing antibodies, but sensitivity varies by subtype. AIDS 27, 1535-1544 (2013).

4. Rusert, P. et al. Determinants of HIV-1 broadly neutralizing antibody induction. Nat. Med. 22, 1260-1267 (2016).

5. Kadelka, C. et al. Distinct, IgG1-driven antibody response landscapes demarcate individuals with broadly HIV-1 neutralizing activity. J. Exp. Med. 215, 15891608 (2018)

6. Shankarappa, R. et al. Consistent viral evolutionary changes associated with the progression of human immunodeficiency virus type 1 infection. J. Virol. $\mathbf{7 3}$ 10489-10502 (1999).

7. Bertels, F. et al. Dissecting HIV virulence: heritability of setpoint viral load, CD4 T cell decline and per-parasite pathogenicity. Mol. Biol. Evol. 35, 27-37 (2018).

8. Blanquart, F. et al. Viral genetic variation accounts for a third of variability in HIV1 set-point viral load in Europe. PLoS Biol. 15, e2001855 (2017).

9. McLaren, P. J. et al. Polymorphisms of large effect explain the majority of the host genetic contribution to variation of HIV-1 virus load. Proc. Natl Acad. Sci. USA 112, 14658-14663 (2015).

10. Bhiman, J. N. et al. Viral variants that initiate and drive maturation of V1V2-directed HIV-1 broadly neutralizing antibodies. Nat. Med. 21, 1332-1336 (2015).

11. Simonich, C. A. et al. HIV-1 neutralizing antibodies with limited hypermutation from an infant. Cell 166, 77-87 (2016).

Acknowledgements Financial support for this study has been provided by the Swiss National Science Foundation (SNF; 314730_152663 and 314730_172790 to A.T.; 324730B_179571 to H.F.G.; PZOOP3-142411 and BSSGIO 155851 to R.D.K.), the Clinical Priority Research Program of the University of Zurich (Viral infectious diseases: Zurich Primary HIV Infection Study to H.F.G. and A.T.), the Yvonne-Jacob Foundation (to H.F.G.), the Swiss 
Vaccine Research Institute (to A.T., H.F.G. and R.D.K.) and the SystemsX.ch grant AntibodyX (to A.T.). This study has been cofinanced within the framework of the Swiss HIV Cohort Study, supported by the SNF (33CS30_148522 to H.F.G.), by the small nested SHCS project 744 (to A.T.) and by the SHCS research foundation. The funders had no role in study design, data collection and analysis, decision to publish, or preparation of the manuscript. The SHCS data are collected by the five Swiss University Hospitals, two Cantonal Hospitals, 15 affiliated hospitals and 36 private physicians (listed in http:// www.shcs.ch/180-health-care-providers). We thank the patients participating in the ZPHI and the SHCS and their physicians and study nurses for patient care and D. Perraudin and M. Minichiello for administrative assistance.

Reviewer information Nature thanks P. Lemey, J. Overbaugh and the other anonymous reviewer(s) for their contribution to the peer review of this work.

Author contributions R.D.K., P.R., C.K., H.F.G. and A.T. conceived and designed the study and analysed data. M.Hu., A.M., H.E., M.S., T.L., N.F., J.W., T.U., N.S.B., C.L., H.K., J.B. and K.J.M. conducted experiments and analysed data. D.L.B., A.U.S., J.-P.C., M.C., E.B., M.Ho., A.C., M.B., A.R., S.Y., V.A., T.K., H.F.G. and the members of the Swiss HIV Cohort Study conceived and managed the SHCS and ZPHI cohorts, collected and contributed patient samples and clinical data. R.D.K., C.K., M.S., H.F.G. and A.T. wrote the manuscript, on which all co-authors commented.

Competing interests The University of Zurich filed a European patent application (EP18184854.0) that includes the full envelope sequences of patients T282 and R282 or components thereof for use as bNAb-inducing immunogens with R.D.K., P.R., H.F.G. and A.T. listed as inventors. All other authors declare no competing interests.

Additional information

Extended data is available for this paper at https://doi.org/10.1038/s41586018-0517-0.

Supplementary information is available for this paper at https://doi.org/ 10.1038/s41586-018-0517-0.

Reprints and permissions information is available at http://www.nature.com/ reprints.
Correspondence and requests for materials should be addressed to R.D.K. or H.F.G. or A.T.

Publisher's note: Springer Nature remains neutral with regard to jurisdictional claims in published maps and institutional affiliations.

\section{The Swiss HIV Cohort Study}

Alexia Anagnostopoulos ${ }^{2}$, Manuel Battegay ${ }^{8}$, Enos Bernasconi ${ }^{5}$, Jürg Böni ${ }^{1}$, Dominique L. Braun ${ }^{2}$, Heiner C. Bucher ${ }^{17}$, Alexandra Calmy ${ }^{7}$.

Matthias Cavassini ${ }^{4}$, Angela Ciuffi ${ }^{18}$, Günter Dollenmaier ${ }^{19}$, Matthias Egger ${ }^{20}$ Luigia Elzi ${ }^{8}$, Jan Fehr ${ }^{2}$, Jacques Fellay ${ }^{21}$, Hansjakob Furrer ${ }^{9}$, Christoph A. Fux ${ }^{22}$, Huldrych F. Günthard ${ }^{2}$, David Haerry ${ }^{23}$, Barbara Hasse ${ }^{2}$, Hans H. Hirsch ${ }^{8,12}$, Matthias Hoffmann ${ }^{6}$, Irene Hösli24, Michael Huber ${ }^{1}$, Christian Kahlert ${ }^{6,25}$, Laurent Kaiser ${ }^{10}$, Olivia Keiser ${ }^{26}$, Thomas Klimkait ${ }^{12}$, Roger D. Kouyos ${ }^{2}$, Helen Kovari², Bruno Ledergerber ${ }^{2}$, Gladys Martinetti ${ }^{27}$,

Begona Martinez de Tejada ${ }^{28}$, Catia Marzolini ${ }^{8}$, Karin J. Metzner², Nicolas Müller ${ }^{2}$, Dunja Nicca ${ }^{6}$, Paolo Paioni ${ }^{29}$, Guiseppe Pantaleo ${ }^{11}$, Matthieu Perreau ${ }^{11}$, Andri Rauch ${ }^{9}$, Christoph Rudin ${ }^{30}$, Alexandra U. Scherrer ${ }^{1,2}$, Patrick Schmid ${ }^{6}$, Roberto Speck ${ }^{2}$, Marcel Stöckle ${ }^{8}$, Philip Tarr ${ }^{31}$,

Alexandra Trkola ${ }^{1}$, Pietro Vernazza ${ }^{6}$, Gilles Wandeler ${ }^{9}$, Rainer Weber ${ }^{2}$ \& Sabine Yerly ${ }^{10}$

${ }^{17}$ Basel Institute for Clinical Epidemiology and Biostatistics, University Hospital Basel, University of Basel, Basel, Switzerland. ${ }^{18}$ Institute of Microbiology, University Hospital Lausanne, University of Lausanne, Lausanne, Switzerland. ${ }^{19}$ Centre for Laboratory Medicine, Canton St. Gallen, St. Gallen, Switzerland. ${ }^{20}$ Institute of Social and Preventive Medicine, University of Bern, Bern, Switzerland. ${ }^{21}$ Global Health Institute, School of Life Sciences, Ecole Polytechnique Fédérale de Lausanne, Lausanne, Switzerland. ${ }^{22}$ Clinic for Infectious Diseases and Hospital Hygiene, Kantonsspital Aarau, Aarau, Switzerland. ${ }^{23}$ Positive Council, Zurich, Switzerland. ${ }^{24}$ Clinic for Obstetrics, University Hospital Basel, University of Basel, Basel, Switzerland. ${ }^{25}$ Children's Hospital of Eastern Switzerland, St. Gallen, Switzerland. ${ }^{26}$ Institute of Global Health, University of Geneva, Geneva, Switzerland. ${ }^{27}$ Cantonal Institute of Microbiology, Bellinzona, Bellinzona, Switzerland. ${ }^{28}$ Department of Obstetrics and Gynecology, University Hospital Geneva, University of Geneva, Geneva, Switzerland. ${ }^{29}$ University Children's Hospital, University of Zurich, Zurich, Switzerland. ${ }^{30}$ University Children's Hospital, University of Basel, Basel, Switzerland. ${ }^{31}$ Kantonsspital Baselland, University of Basel, Basel, Switzerland 


\section{METHODS}

Data reporting. No statistical methods were used to predetermine sample size. The experiments were not randomized. The investigators were blinded to allocation (transmitter-recipient pairing) during initial antibody response analyses (all binding antibody responses and neutralization analyses to an eight-virus multi-clade panel. The investigators were not blinded during outcome assessment and extended neutralization analyses.

Study populations and ethics information. The starting cohort for our analysis were 4,281 chronic HIV-1-infected individuals included in a population-wide screen for HIV-1 neutralization breadth, now termed the Swiss 4.5K Screen ${ }^{4}$, for which extensive plasma neutralization and HIV-1 binding data were generated in a separate study ${ }^{5}$ (Extended Data Fig. 1a). Detailed information on the plasma sample/patient selection and study design of the Swiss $4.5 \mathrm{~K}$ Screen has been previously described ${ }^{4}$ (Extended Data Fig. 1a). All plasma samples were collected during viraemic periods (no antiretroviral treatment at sampling time point) from adult, chronically infected HIV-1 patients. Because the cross-sectional sample selection was pre-determined by the Swiss $4.5 \mathrm{~K}$ Screen, it was not possible during our current study to select samples matched for infection length or for closeness to putative transmission time points. Important for the interpretation of specific findings in the current study, the study design of the Swiss $4.5 \mathrm{~K}$ Screen included three patient groups that differed in infection length ( $1-3,3-5$ and $\geq 5$ years of untreated HIV-1 infection). The highest frequency of neutralization breadth was observed among individuals infected for $>3$ years ${ }^{4}$. The plasma samples analysed previously $y^{4,5}$ and in the current study were provided by the biobanks of the Swiss HIV Cohort study (SHCS) and the Zurich Primary HIV Infection Study (ZPHI).

The SHCS and the ZPHI have been approved by the ethics committee of the participating institutions (Kantonale Ethikkommission Bern, Ethikkommission des Kantons St. Gallen, Comite departemental d'ethique des specialites medicales et de medicine communataire et de premier recours, Hôpitaux Cantonale de Genève, Kantonale Ethikkommission Zürich, Repubblica e Cantone TicinoComitato Ethico Cantonale, Commission cantonale d'étique de la recherche sur l'être humain, Canton de Vaud, Lausanne, Ethikkommission beider Basel for the SHCS and Kantonale Ethikkommission Zürich for the ZPHI) and written informed consent had been obtained from all participants. Please see the supplementary note of the previous study ${ }^{4}$ and the Supplementary Methods of the current study for further details on the cohorts and the collected patient data. The SHCS is a prospective, nationwide, longitudinal, non-interventional, observational, clinic-based cohort with semi-annual visits and blood collections, enrolling all HIV-infected adults living in Switzerland ${ }^{12}$. Detailed information on the SHCS is available on http://www.shcs.ch. The SHCS, founded in 1988, is highly representative of the HIV epidemic in Switzerland as it includes an estimated $53 \%$ of all HIV cases diagnosed in Switzerland since the onset of the epidemic, $72 \%$ of all patients receiving antiretroviral treatment in Switzerland, and 69\% of the nationwide-registered AIDS cases $^{12}$. The SHCS is registered under the Swiss National Science longitudinal platform: http://www.snf.ch/en/funding/programmes/longitudinal-studies/Pages/ default.aspx\#Currently\%20supported\%20longitudinal\%20studies.

The ZPHI is a continuous, observational, non-randomized, single-centre cohort founded in 2002 that specifically enrols patients with documented acute or recent primary HIV-1 infection (https://www.clinicaltrials.gov/; ID NCT00537966) ${ }^{13}$.

Establishment of the transmission pair cohort. Overview. We established a cohort of 303 putative transmission pairs by screening for potential transmission pairs within a cross-sectional starting cohort of 4,281 chronic HIV-1-infected individuals included in the Swiss $4.5 \mathrm{~K}$ Screen ${ }^{4,5}$. In addition to extensive patient demographic and clinical data, we had access to data on pol sequence, neutralization activity ${ }^{4}$ and HIV-1 binding antibody responses ${ }^{5,14}$ (Extended Data Fig. 1d) for these individuals.

We phylogenetically determined potential transmission pairs within the 4,281 patient starting cohort based on a threshold of pol gene similarity that was allowed to include individuals with a long infection history (Extended Data Fig. 1e, f and Supplementary Data 1). pol similarity has been previously established as reliable method to record genetic similarity of the infecting virus. Almost all larger molecular epidemiology work in HIV is based on HIV pol ${ }^{15-19}$. Moreover, owing to genetic linkage, similarity in pol and similarity in env are highly correlated in transmission pairs ${ }^{13,20,21}$. We determined potential transmission pairs as nearest neighbours (cherries) with a genetic distance of less than 0.045 on a pol phylogeny ${ }^{16,22}$ (Extended Data Fig. 1e, f and Supplementary Data 1). In total, we identified 303 potential transmission pairs and assigned transmitter and recipient status based on estimated infection dates. Individuals in transmission pairs were predominantly male, men who have sex with men and infected with subtype B (Supplementary Data 1 and Extended Data Table 1), reflecting the main drivers of domestic HIV-1 transmission in Switzerland ${ }^{4,12,13}$. Overall, the cohort of 303 transmission pairs did not differ in terms of duration of infection, neutralization breadth, virus load and peripheral CD4 $\mathrm{T}$ cell counts from the remaining patients of the starting cohort (Extended Data Table 1). Specific steps conducted in the establishment of the cohort are described in full in the following sub-sections.
Construction of HIV-1 pol gene phylogenies. Potential transmission pairs were defined based on HIV-1 pol gene phylogenies (as described in detail previously ${ }^{16,22}$ ) using pol nucleotide sequence data available in the SHCS database. In brief, these sequences stem from clinically or epidemiologically implicated genotypic resistance tests and, thus, are not derived from the same sampling time points as the plasma samples used for analyses of antibody responses.

We constructed pol phylogenies from 19,604 partial pol sequences from 10,970 different SHCS cohort participants, which included the 4,281 patients of the starting cohort (Extended Data Fig. 1d), and an additional 90,994 sequences from the Los Alamos database (http://www.hiv.lanl.gov/). These latter were included to decrease the chances of false-positive random clustering. We retrieved from the Los Alamos database all available, non-Swiss, pol sequences (region: 2,253-3,870) with a minimal length of $900 \mathrm{bp}$ as of September 2014. Redundant control sequences (different sequence ID but identical nucleotide sequence) were deduplicated. For the SHCS patients, sequences with a minimum length of $250 \mathrm{bp}$ for the protease gene and $500 \mathrm{bp}$ for the RT gene were included. All sequences were initially aligned to a HXB2 reference genome (http://www.ncbi.nlm.nih.gov/nuccore/K03455.1) using MUSCLE ${ }^{23}$. Next, insertions relative to HXB2 and resistance mutations according to Stanford (http://hivdb.stanford.edu/) and International Antiviral Society-USA (https://www.iasusa.org/) lists were removed. In the following step, a generalized time-reversible model-based tree was constructed using FastTree ${ }^{24}$ The R package 'APE' version 3.1 was used for tree exploration and analysis ${ }^{25}$. Definition of potential transmission pairs. Transmission pairs were defined as monophyletic pairs of the 4,281 starting cohort sequences on the pol phylogeny. We assumed that pairs with a cophenetic distance $>4.5 \%$, a commonly used threshold, clustered due to statistical and/or methodological artefacts (such as underrepresentation or even absence of rare genotypes in the background sequences). These pairs were therefore disregarded. We chose the relatively liberal threshold of pair distances of up to $4.5 \%$ as it best accommodates our cohort and the research questions addressed for several reasons. Owing to the study design of the Swiss $4.5 \mathrm{~K}$ Screen, infection length varied between participants as one intent of this screen was to assess the influence of infection length on antibody development. Additionally, sampling time points for antibody testing and pol sequencing differ as the latter data were retrieved from clinically implicated genotypic resistance tests (see above). Short genetic distances in pol are associated with recent sampling times (Extended Data Fig. 1f). A strict distance criterion therefore bears the increased risk to exclude pairs in which plasma was sampled during prolonged chronic infection (at least 3 years of untreated infection), which we have previously shown to be associated with broad neutralization ${ }^{4}$. Thus, high genetic distances are a marker for the length of the within-patient evolution of the virus and associated with bNAb development, a main outcome of our study. A more liberal distance threshold therefore ascertains that the relevant patient population is included, maximizing the statistical power of our analysis. It is also important to note that, since we included a large number of background sequences, the detected monophyly is already a strong signal for the formation of a pair, irrespective of the actual genetic distance. We nevertheless verified the results of our study in sensitivity analyses using stricter selection criteria for potential transmission pairs, all of which confirmed the robustness of our observations (Extended Data Fig. 5).

Potential and confirmed transmission pairs. The attending physician confirmed that the bNAb transmission pair (T282 and R282) was a heterosexual couple living in a stable relationship in Switzerland, with a female-to-male transmission based on clinical data and self-reporting by the patients. Transmission of a HIV-1 subtype B infection from the female (North African origin) to the male partner (Asian origin) is further supported by a higher genetic pol diversity of the female partner (diversity $=0.53 \%$ for $\mathrm{T} 282$ and diversity $=0.20 \%$ for $\mathrm{R} 282$ on the same sampling date) On the basis of the available demographic data and infection timing, T282 infected R282 around 1.9 years post infection. The analysed plasma samples of the transmitter and recipient were collected 3.8 years and 3.4 years post infection, respectively. With the exception of T282 and R282, all other pairs should be viewed as potential transmission pairs as they possess genetically highly related virus strains in the pol phylogeny but have not been confirmed by alternative measures (physician records, self-reporting, and so on) and thus theoretically could also be partners in a tightly linked transmission cluster involving several people. For brevity we use the collective terms transmission pair(s)/transmission pair cohort without adding potential and/or confirmed to distinguish the respective patients. It is important to note that, as only the genetic relatedness of the infecting virus is of relevance for our study, neither the definition of confirmed pairs nor the tracing of the transmission time point is of relevance in the context of the conducted analyses.

Definition of transmitter and recipient within a pair. In a given transmission pair, we identified the individual with the earlier estimated infection date as the transmitter and the other as the recipient. In accordance with a previous study ${ }^{26}$, our results were, however, not affected by this assumption. The analyses shown in Fig. 1c, Extended Data Figs. 3b, c, 5d, e and Extended Data Table 2 are by necessity symmetrical (that is, insensitive) with respect to which patient is identified 
as transmitter and recipient. The analyses shown in Fig. 2a, b and Extended Data Figs. $4,5 \mathrm{a}-\mathrm{c}$ are not by necessity symmetrical, that is, they are potentially affected by which patient is identified as transmitter and recipient. We obtained, however, identical results when randomly assigning the role of transmitter and recipient within a transmission pair (Extended Data Fig. 3a). Note that this type of randomization does not change the grouping of patients into pairs but only which patient in a pair is considered transmitter or recipient.

HIV-1 binding antibody profile of the transmission pair cohort. The plasma IgG1, IgG2, IgG3 binding antibody reactivity to 13 HIV-1 Gag and Env antigens have been established for all 606 individuals in the 303 transmission pair cohort in a prior study analysing HIV-1 binding antibody of the 4,281 starting cohort ${ }^{5}$. See Supplementary Methods for details.

HIV-1 neutralizing antibody profile of the transmission pair cohort. Plasma neutralization activity of the 606 patients in the transmission pair cohort against a 14 multi-clade pseudovirus panel was determined as part of the current study. See Supplementary Methods for details.

Profiling of the neutralization breadth in the bNAb transmission pair T282 and R282. See Supplementary Methods.

Prediction of bNAb epitope specificity in plasma bNAb of transmission pair T282 and R282. See Supplementary Methods and a previously published study ${ }^{27}$. Strategies to determine the antibody-imprinting capacity of HIV-1. To investigate the similarity of antibody responses in transmission pairs, we used a series of approaches (summarized in Extended Data Fig. 2e and detailed below). In the context of our study, the strength of the overall effect of virus genetics on antibody responses measured by the above analyses is of secondary importance, as it can be the result of a strong influence of virus genetics in a few transmission cases and a weak/non-existent influence in most other cases within the cohort. Our analyses are thus tailored to provide proof of existence of an overall viral heritability, which-even if low-is a necessary condition for the search for bNAb imprinter viruses in a next step. The essence of what we investigate (see Fig. 1) is thus the similarity of the pattern of the responses across antigens/viruses and not that specific individuals have high antibody responses to all antigens/viruses.

Our analysis strategy followed three principle steps. First, we tested, for all 14 neutralization and 39 binding parameters, whether a given parameter is correlated within transmission pairs using Spearman correlations ( $\rho_{\text {Spearman }}$ for each parameter) (Fig. 1a and Extended Data Figs. 3a, 4a, c).

Second, the strength of the association is averaged across all neutralization, IgG1, IgG2 or IgG3 parameters to obtain a measure for the overall similarity of responses (average $\rho_{\text {Spearman; }}$ Fig. $1 \mathrm{~b}$ and Extended Data Figs. $4 \mathrm{~b}, \mathrm{~d}, 5 \mathrm{a}-\mathrm{c}$ ).

Third, a mixed-effect Tobit model was used to estimate the fraction of the variance explained by viral genetic factors, which can in addition be adjusted for the effect of covariables (Fig. 1c and Extended Data Figs. 3b, c, 5d, e). A Tobit model was used because the neutralization percentage data are always non-negative and the binding data are approximately uniformly distributed within the range of $0-1$. The 303 pairs constitute the groups used in the mixed-effect Tobit model. The model estimates three types of variances, the group-level variance $\sigma_{\mathrm{P}}{ }^{2}$, the individual level variance $\sigma_{1}^{2}$, and (if cofactors are included in the model) the variance explained by the cofactors $\sigma_{\mathrm{C}}{ }^{2}$. Accordingly, the heritability is then given by

$$
h=\frac{\sigma_{\mathrm{P}}^{2}}{\sigma_{\mathrm{P}}^{2}+\sigma_{\mathrm{I}}^{2}+\sigma_{\mathrm{C}}^{2}}
$$

This constitutes a conservative approach to estimate heritability, since the variance explained by cofactors is also included in the denominator; that is, it is assumed to contribute to the non-heritable portion of the response although some cofactors (viral load, subtype and diversity) are at least partially steered by virus genetics. This approach is applied to each pseudovirus of the neutralization panel and each antigen separately and then averaged over all pseudoviruses/antigens in order to obtain an overall heritability estimate (Fig. 1c, Extended Data Figs. 3b, c, 5d, e and Extended Data Table 2).

In addition, we used linear mixed-effect models as an alternative method to derive heritability estimates, as these are commonly used $\mathrm{d}^{26,28}$ and faster to be computed than Tobit models. The resulting estimates from variously adjusted linear mixed-effect models as well as standard errors derived from these models (by leave-one-out analyses) are compared to the Tobit estimates in Extended Data Fig. 3b. The standard errors proved to be small, and the linear mixed-effect models revealed almost identical effects to the Tobit mixed-effect models, highlighting the robustness of our findings.

In all three approaches, the statistical significance of a given heritability value (either for an individual pseudovirus or antigen, or averaged across pseudoviruses/ antigens) is derived by comparing the observed value with the distribution obtained in 1,000 random reassignments of the recipients. The respective $P$ value is then given by the inverse of the number of randomizations for which heritability is at least as high as in the original dataset. In Fig. $1 \mathrm{~b}$ and Extended Data Figs. 4b, d,
$5 \mathrm{a}-\mathrm{c}$, we consider two kinds of random reassignments: (1) completely random reassignment of recipients to transmitters and (2) random reassignment of recipients to transmitters with the same demographics (subtype (B versus non- $B$ ), ethnicity (white versus non-white), untreated infection length (1-3 years, 3-5 years, $>5$ years)). The latter adjusts for the potentially confounding effect of subtype, ethnicity and infection length. See also Supplementary Methods and previously published studies ${ }^{29,30}$ for details on how we controlled for potential confounders. Single-genome amplification of the HIV-1 envelope. See Supplementary Methods and a previous study ${ }^{31}$.

HIV-1 envelope cloning from undiluted cDNA. See Supplementary Methods. Infusion vector cloning and sequencing. See Supplementary Methods.

HIV-1 full-length genome sequencing and analysis. See Supplementary Methods and previously published studies ${ }^{32-36}$.

HIV-1 envelope phylogenetic analysis. See Supplementary Methods and previously published studies ${ }^{37-39}$.

Systematic strategy to trace HIV-1 strains with bNAb-imprinting capacity. To derive a method that allows a systematic identification of HIV-1 strains that have the capacity to induce (imprint) bNAb activity, we used a multi-step approach summarized in Extended Data Fig. 8.

Step 1. For each transmission pair, we test whether the antibody response across pseudo-viruses or antigens is correlated. This indicates whether the two members of a pair have high responses against the same viruses or antigens. Specifically, we determine the Spearman correlation coefficient of the neutralization responses in transmitter and recipient, and the same is done for the IgG1-binding response for each transmission pair. The two correlation coefficients are combined to obtain a single antibody response similarity index $\left(1 / 2 \rho_{\text {Spearman-neutralization }}+1 / 2 \rho_{\text {Spearman-IgG1-binding }}\right)$. Note, we only use IgG1 binding responses as these dominate natural HIV-1 infections and also provided the highest within-pair similarity (average $\rho_{\text {Spearman }}=0.19, P_{\text {shuffling }}<0.001$; Fig. 1a, b).

Step 2. Because a large fraction of patients exhibits weak responses against all tested pseudoviruses ( 148 out of 606 patients distributed over 127 pairs neutralize each of the 14 viruses at $<20 \%$, that is, neutralization score 0 ), and the comparison of their fingerprints is thus uninformative, this fingerprint-based approach had to be restricted to pairs in which both patients exhibit a certain level of neutralization strength (as measured by the neutralization score). For each transmission pair, we calculated the neutralization strength as the minimum neutralization score of the two individuals.

Step 3. Transmission pairs with highly similar antibody binding responses and high neutralization scores can now be identified (Fig. 3a, b).

Step 4 . The number of transmission pairs with a similar (binding) and strong (neutralization) antibody response is determined for a broad range of similarity $(0.3,0.4,0.5$ and 0.6$)$ and strength thresholds $(1,2, \ldots, 7)$. To determine significance, these numbers were compared to those observed in 1,000 replicate datasets with shuffled transmission pairs, for which the shuffling is realized by assigning a randomly chosen recipient (sampling without replacement) to each transmitter (Fig. 3c, d).

For the interpretation of the results, it is important to note that not all pairs can be expected to show similar responses as in particular neutralization breadth is genuinely rare. In addition, in cross-sectional analyses as ours, it must be anticipated that occasionally the effects of the infecting virus are masked by the effect of differential infection length or other confounding factors in transmitter and recipient. We therefore control intensely for confounding factors (see also section 'Strategies to determine the antibody-imprinting capacity of HIV-1'). The clear overall shift towards positively correlated responses (Fig. 3a, b), which is not a chance finding as we show (Fig. 3c, d), highlights that a considerable fraction of viruses exists that induce similar responses. Among these, rare cases with bNAb imprinting capacity are expected and can be screened for.

Reporting summary. Further information on research design is available in the Nature Research Reporting Summary linked to this paper.

Data availability. The antibody response and patient data reported in this paper are completely tabulated in Supplementary Data 1,2. Sequence data of T282R282 Env consensus and Env clones reported in Fig. 2c are deposited in GenBank. Accession codes are listed in Extended Data Fig. 6b. The raw sequencing files of the Illumina full HIV sequencing data of patients T282 and R282 referred to in Fig. 2c and Extended Data Fig. 6 have been uploaded to https://zenodo.org/ (https://doi. org/10.5281/zenodo.1324259). pol sequence data of the 606 studied cases are available from the corresponding authors and/or the SHCS scientific board (http://www. shcs.ch/contact) upon request.

12. Schoeni-Affolter, F. et al. Cohort profile: the Swiss HIV Cohort study. Int. J. Epidemiol. 39, 1179-1189 (2010).

13. Rieder, P. et al. Characterization of human immunodeficiency virus type 1 (HIV-1) diversity and tropism in 145 patients with primary HIV-1 infection. Clin. Infect. Dis. 53, 1271-1279 (2011). 
14. Liechti, T. et al. Development of a high-throughput bead based assay system to measure HIV-1 specific immune signatures in clinical samples. J. Immunol. Methods 454, 48-58 (2018).

15. von Wyl, V. et al. The role of migration and domestic transmission in the spread of HIV-1 non-B subtypes in Switzerland. J. Infect. Dis. 204, 1095-1103 (2011).

16. Kouyos, R. D. et al. Molecular epidemiology reveals long-term changes in HIV type 1 subtype B transmission in Switzerland. J. Infect. Dis. 201, 1488-1497 (2010).

17. Brenner, B., Wainberg, M. A. \& Roger, M. Phylogenetic inferences on HIV-1 transmission: implications for the design of prevention and treatment interventions. AIDS 27, 1045-1057 (2013).

18. Wertheim, J. O. et al. The global transmission network of HIV-1. J. Infect. Dis. 209, 304-313 (2014).

19. Hassan, A. S., Pybus, O. G., Sanders, E. J., Albert, J. \& Esbjörnsson, J. Defining HIV-1 transmission clusters based on sequence data. AIDS 31, 1211-1222 (2017).

20. Hué, S., Clewley, J. P., Cane, P. A. \& Pillay, D. HIV-1 pol gene variation is sufficient for reconstruction of transmissions in the era of antiretroviral therapy. AIDS 18 719-728 (2004).

21. Oberle, C. S. et al. Tracing HIV-1 transmission: envelope traits of HIV-1 transmitter and recipient pairs. Retrovirology 13, 62 (2016).

22. Marzel, A. et al. HIV-1 transmission during recent infection and during treatment interruptions as major drivers of new infections in the Swiss HIV Cohort Study. Clin. Infect. Dis. 62, 115-122 (2016).

23. Edgar, R. C. MUSCLE: multiple sequence alignment with high accuracy and high throughput. Nucleic Acids Res. 32, 1792-1797 (2004).

24. Price, M. N., Dehal, P. S. \& Arkin, A. P. FastTree: computing large minimum evolution trees with profiles instead of a distance matrix. Mol. Biol. Evol. 26 , 1641-1650 (2009).

25. Paradis, E., Claude, J. \& Strimmer, K. APE: analyses of phylogenetics and evolution in R language. Bioinformatics 20, 289-290 (2004).
26. Bachmann, N. et al. Parent-offspring regression to estimate the heritability of an HIV-1 trait in a realistic setup. Retrovirology 14, 33 (2017).

27. Tiller, T. et al. Efficient generation of monoclonal antibodies from single human B cells by single cell RT-PCR and expression vector cloning. J. Immunol. Methods 329, 112-124 (2008).

28. Mitov, V. \& Stadler, T. A practical guide to estimating the heritability of pathogen traits. Mol. Biol. Evol. 35, 756-772 (2018)

29. Venner, C. M. et al. Infecting HIV-1 subtype predicts disease progression in women of sub-Saharan Africa. EBioMedicine 13, 305-314 (2016).

30. Alizon, S. \& Fraser, C. Within-host and between-host evolutionary rates across the HIV-1 genome. Retrovirology 10, 49 (2013).

31. Salazar-Gonzalez, J. F. et al. Deciphering human immunodeficiency virus type 1 transmission and early envelope diversification by single-genome amplification and sequencing. J. Virol. 82, 3952-3970 (2008).

32. Giallonardo, F. D. et al. Full-length haplotype reconstruction to infer the structure of heterogeneous virus populations. Nucleic Acids Res. 42, e115 (2014).

33. Gall, A. et al. Universal amplification, next-generation sequencing, and assembly of HIV-1 genomes. J. Clin. Microbiol. 50, 3838-3844 (2012).

34. Zerbino, D. R. \& Birney, E. Velvet: algorithms for de novo short read assembly using de Bruijn graphs. Genome Res. 18, 821-829 (2008)

35. Garrison, E. \& Marth, G. Haplotype-based variant detection from short-read sequencing. Preprint at https://arxiv.org/abs/1207.3907 (2012).

36. Wilm, A. et al. LoFreq: a sequence-quality aware, ultra-sensitive variant caller for uncovering cell-population heterogeneity from high-throughput sequencing datasets. Nucleic Acids Res. 40, 11189-11201 (2012).

37. Camacho, C. et al. BLAST+: architecture and applications. BMC Bioinformatics 10, 421 (2009).

38. Eddy, S. R. Profile hidden Markov models. Bioinformatics 14, 755-763 (1998),

39. Stamatakis, A. RAxML version 8: a tool for phylogenetic analysis and post-analysis of large phylogenies. Bioinformatics 30, 1312-1313 (2014). 


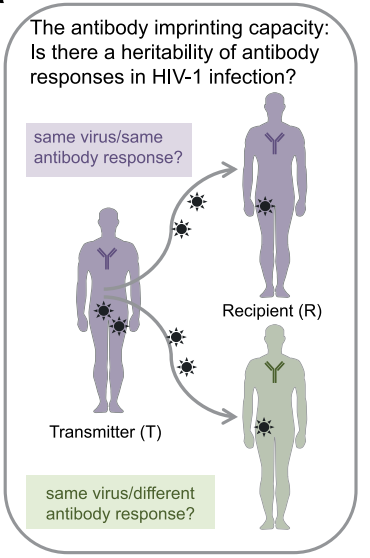

b Does the imprinting capacity of HIV strains differ?

Can bnAb-imprinter strains be identified?

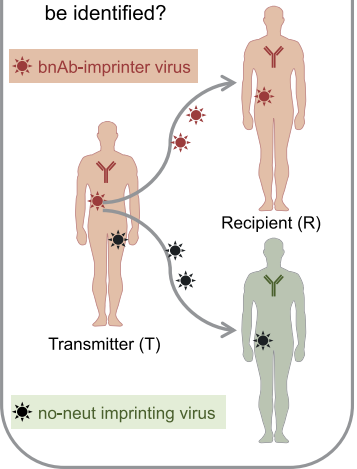

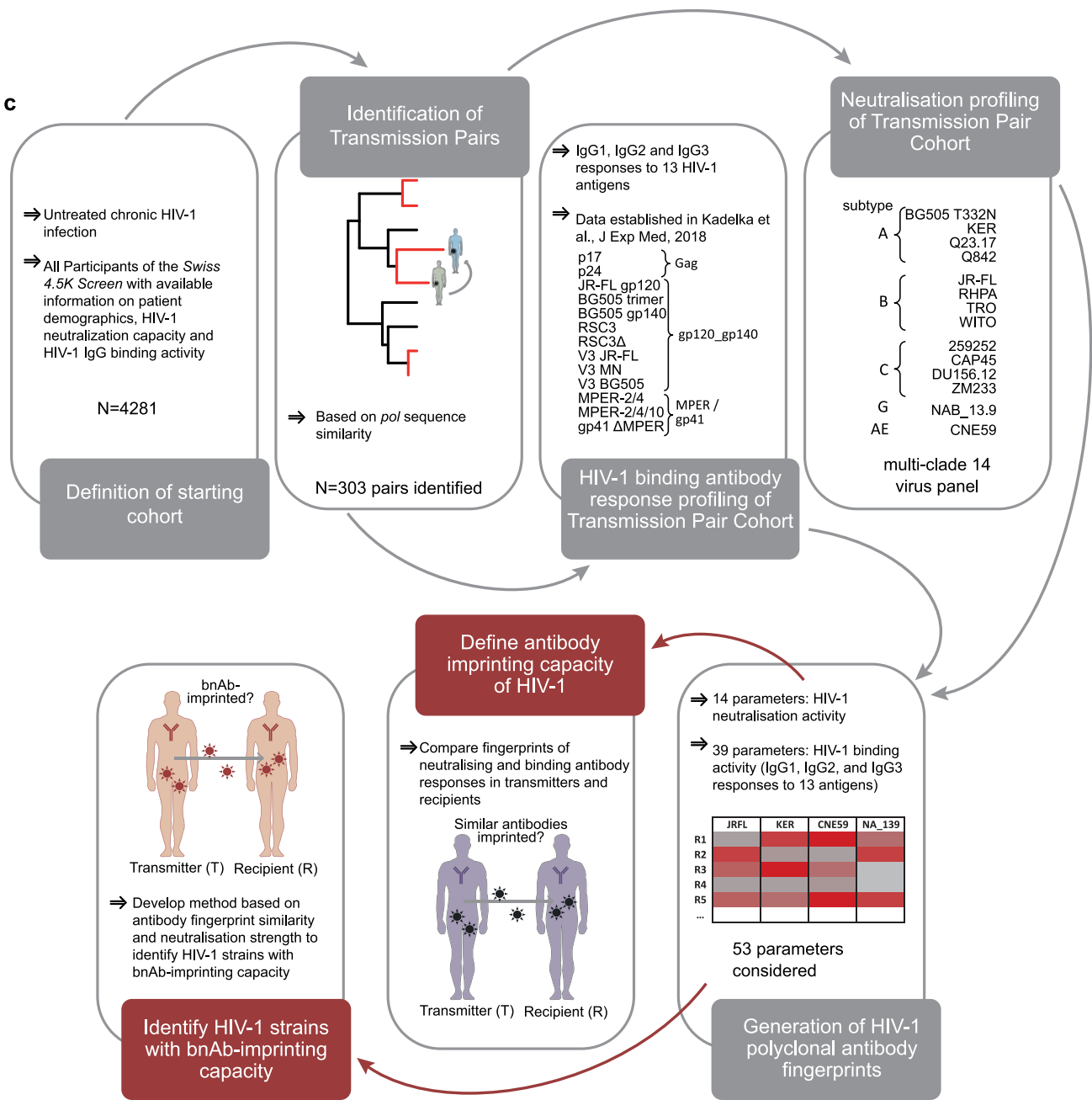

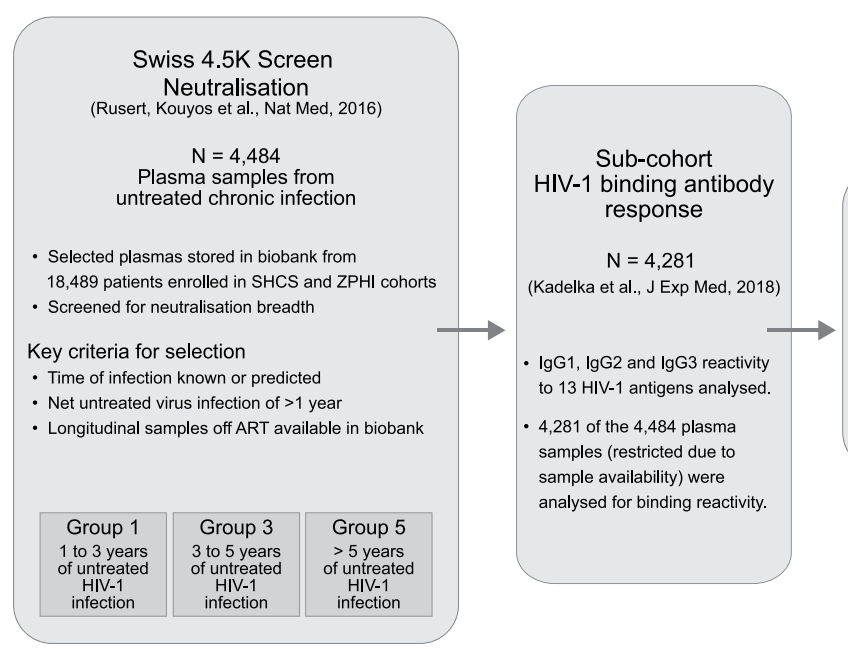

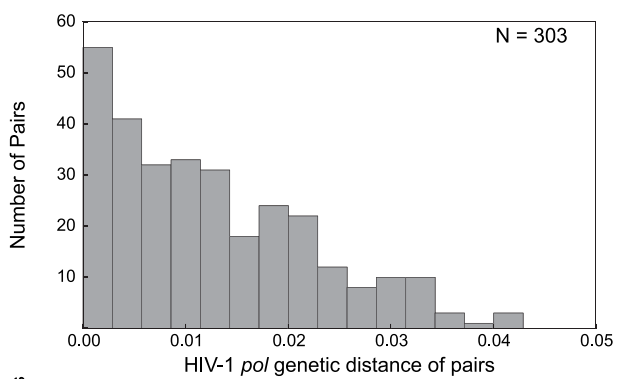

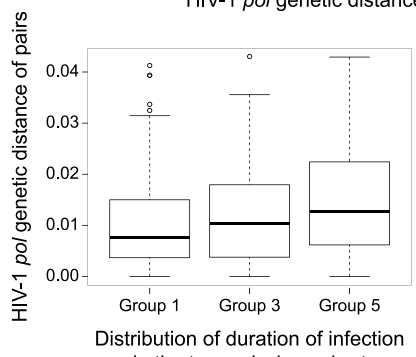

in the transmission cohort

Extended Data Fig. 1 | See next page for caption. 
Extended Data Fig. 1 | Selection and characterization of the HIV-1 transmission cohort used to investigate the antibody imprinting capacity of HIV-1. a, b, Principal aims of the study. a, Defining the binding and neutralizing antibody imprinting capacity of HIV-1 strains/ immunogens (that is, determining the heritability of these responses). The schematic depicts the possible outcomes of a transmission. The recipient may either have the same or a different type of antibody response as the transmitter. b. Creating means to identify bNAb-imprinting HIV-1 strains. The schematic depicts the possible outcomes of a transmission if the transmitter has a bNAb response. The recipient may also develop a bNAb response or not. c, Workflow of the study. d, The search for transmission pairs started with a cohort of 4,281 patients, who were included in the Swiss $4.5 \mathrm{~K} \mathrm{Screen}{ }^{4}$ and for which HIV-1 antibody-binding data are available ${ }^{5}$ e, Histogram of the genetic ( $\left.p o l\right)$ distance of the 303 identified transmission pairs. f, Genetic ( $p o l)$ distance to the transmission partner stratified by duration of untreated infection for the $n=606$ patients in the transmission pair cohort. As in the Swiss $4.5 \mathrm{~K}$ Screen, patients are grouped by infection length into categories of $1-3$ years $(n=138)$, $3-5$ years $(n=252)$ and more than 5 years $(n=216)$. Medians are shown (centre line), each box spans the IQR and each whisker extends to the most extreme value no more than $1.5 \times \mathrm{IQR}$ from the box. More extreme values are shown as points. 
a

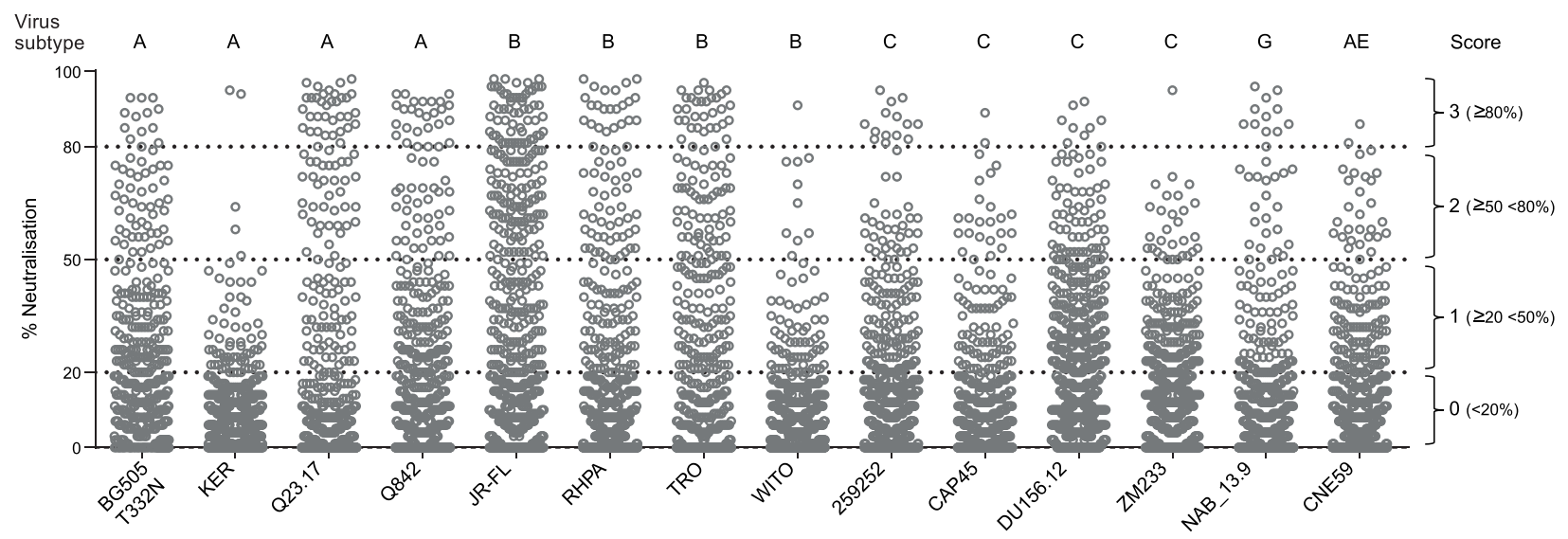

b

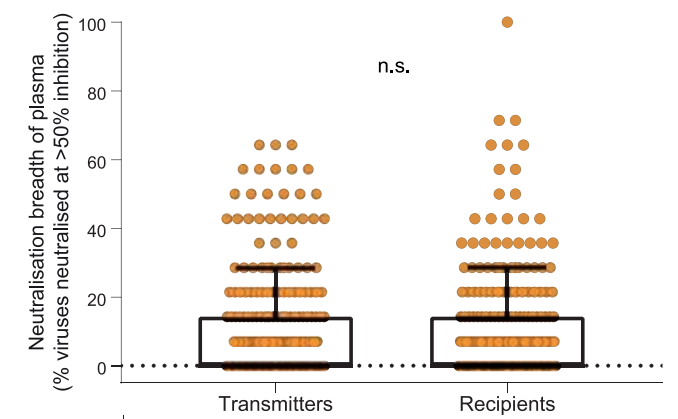

c
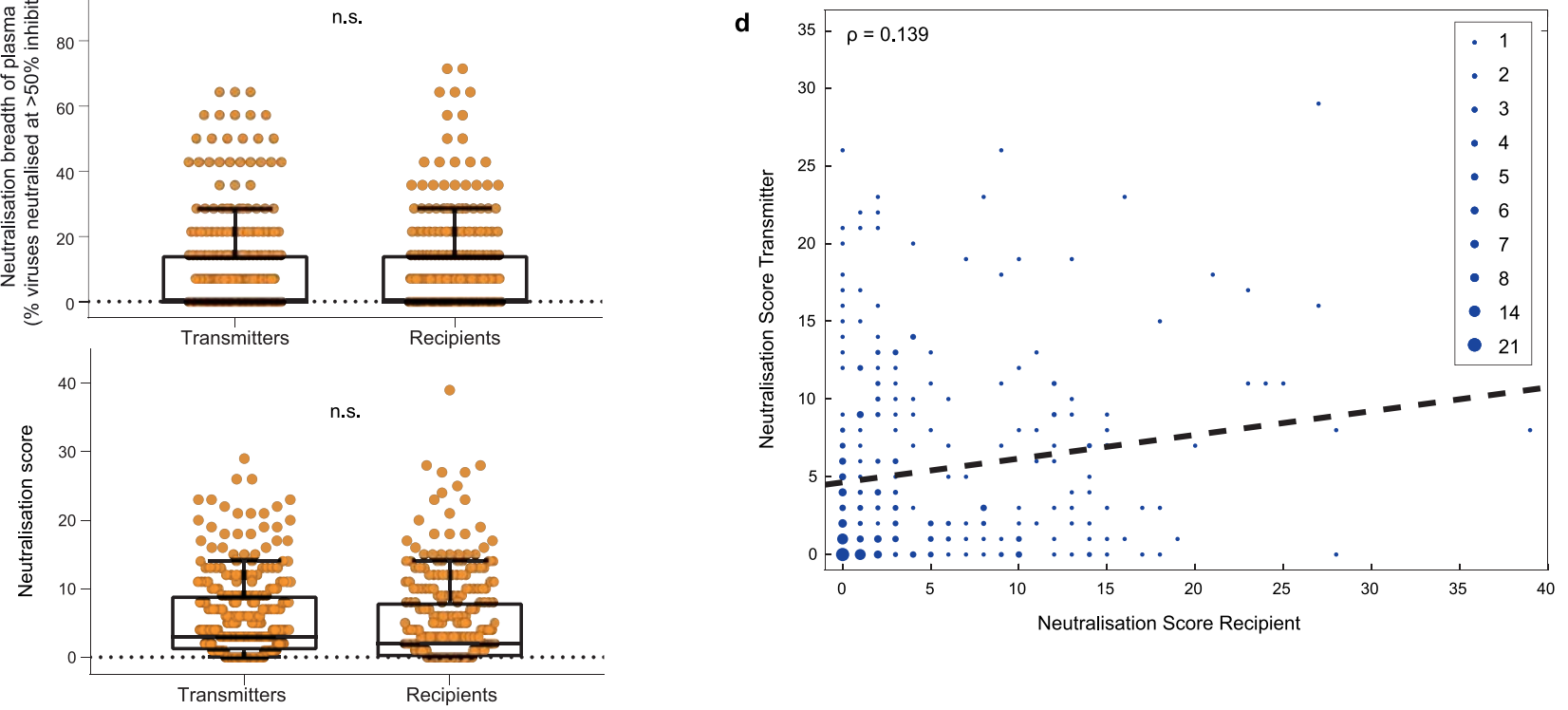

e

Neutralisation

(14 reactivities; recorded as average \% neutralisation at fixed plasma dilution)
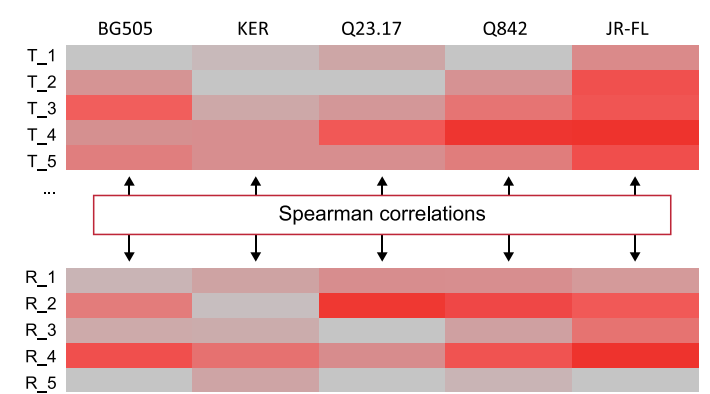

53 Antibody Parameters

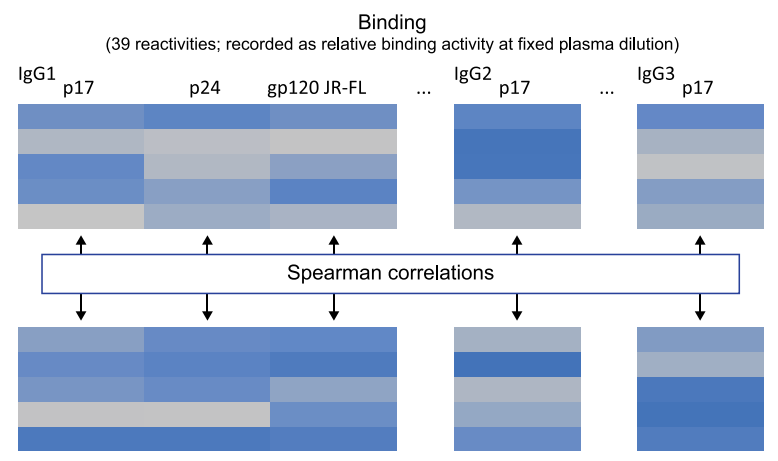

$\downarrow$

Derive average Spearman correlation for all neutralisation responses and all $\operatorname{lgG} 1, \lg \mathrm{G} 2$ and $\lg \mathrm{G} 3$ responses (Fig. 1b and Extended Data Figs. 4b,d,

Mixed-effect Tobit model to estimate impact of infecting virus strain on neutralisation response, IgG1, IgG2 or IgG3 responses (Fig. 1c Extended Data Figs. 3b,c, 5d,e, and Extended Data Table 2).

Derive Spearman correlation for each individual neutr
parameter (Fig. 1a and Extended Data Figs. 3a $4 \mathrm{a}$ )

Extended Data Fig. 2 | Neutralization score analyses and strategies to determine the antibody-imprinting capacity of HIV-1. a, Neutralization activity against a multi-clade 14-virus panel of all 606 transmitter and recipient plasma samples. Dashed horizontal lines at 20\%,50\% and $80 \%$ and brackets display the thresholds used for the assignment of scores. On the basis of these data, each plasma-virus combination received a score of $0-3$. The neutralization score of a plasma sample is the sum of the 14 individual scores against the panel viruses $(0-42)$. Individual data points are shown as jittered circles. b, c, Distribution of neutralization breadth (b; $P=0.69)$ and neutralization scores $(\mathbf{c} ; P=0.40)$ in $n=303$ transmitters and recipients ( $P$ values: two-tailed Wilcoxon signed-rank test). Medians are shown (centre line), each box spans the IQR and the whiskers extend to the $10 \%$ and $90 \%$ percentile. Individual data points are shown as jittered circles. d, Scatter plot of the neutralization score of recipients and transmitters for all 303 transmission pairs (Spearman; $\rho=0.139$, $P=0.015)$. The number of pairs with the indicated values is depicted by dot size. e, Strategies to determine the antibody-imprinting capacity of HIV-1. For each of the 14 neutralization and 39 binding reactivities, the similarity in reactivity (Spearman correlation) was compared between transmitters and recipients to test whether transmission partners shared similar antibody responses. 
a
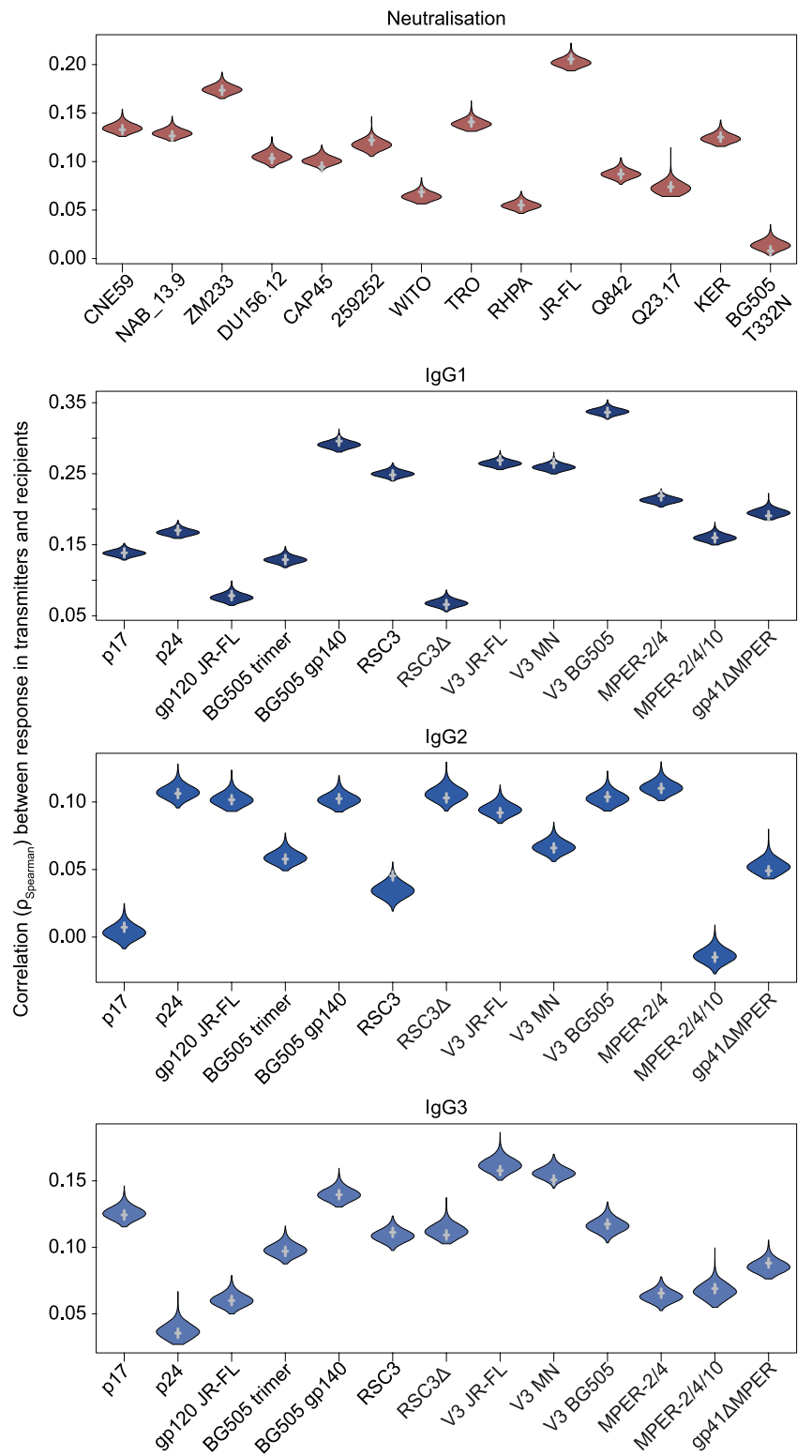

Extended Data Fig. 3 | Antibody similarity measurements are insensitive to the assignment of transmitter and recipient status, to choice of confounders and outliers. a, Distribution of Spearman correlations (as in Fig. 1a) of neutralization-antibody-binding responses in pairs, in which the role of transmitter and recipient was randomly assigned within each of the $n=303$ pairs (1,000 reassignments). Each violin plot is smoothed using a normal kernel, and its width represents the likelihood of a certain Spearman correlation. Grey daggers correspond to the similarities retrieved for the actual assignment of transmitter and recipient used in this study. For all investigated parameters, there was no statistically significant difference between the actual assignment and the shuffled assignment of transmitters and recipients (two-sided $\left.P_{\text {Spearman }}>0.05\right)$. b. Proportion of variability in responses explained b

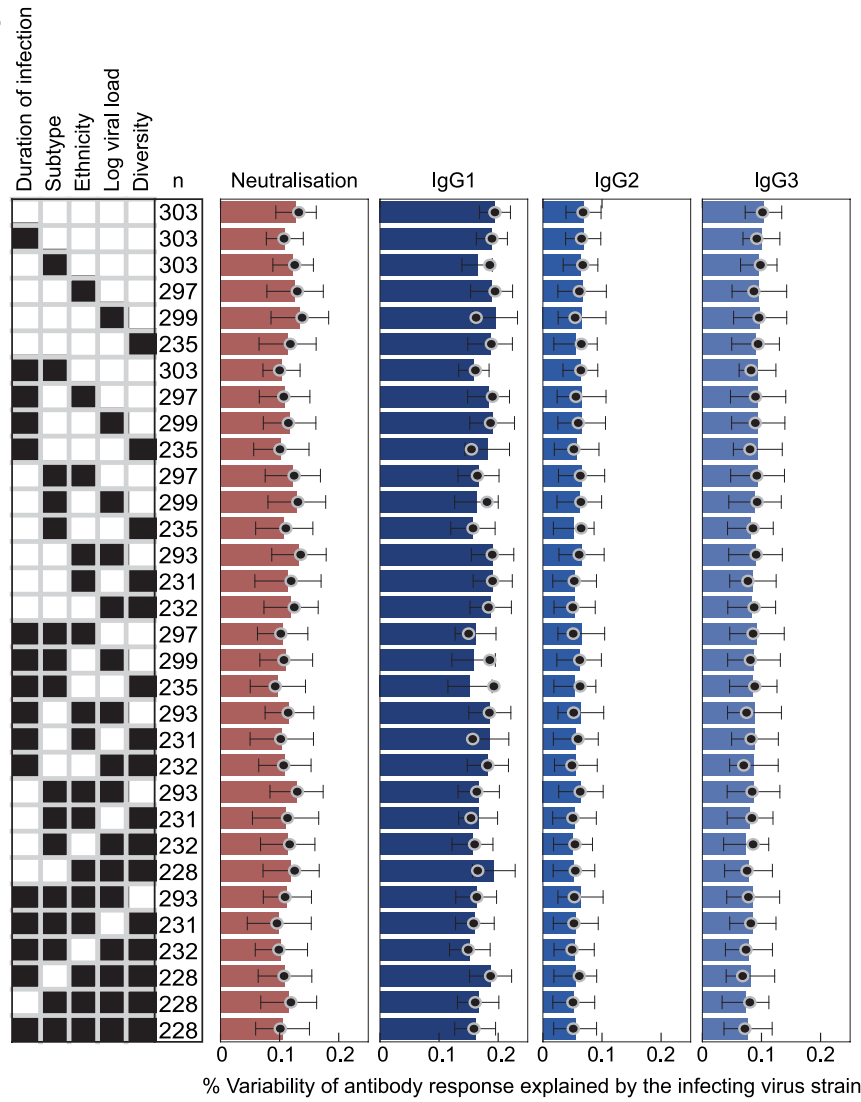

c

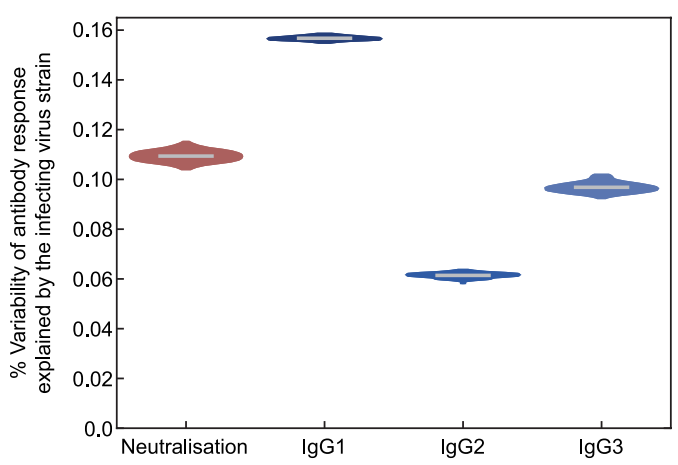

by the infecting virus, determined using mixed-effect Tobit regression models (black circles) and linear mixed-effect models (horizontal bars). Standard errors of the linear mixed-effect models (black error bar; obtained by leave-one-out analyses) are shown. In each row, the models were adjusted for the indicated set of cofactors and only transmission pairs with complete information were included (number of pairs shown per row). c, Proportion of variability in responses explained by the infecting virus, determined using mixed-effect Tobit regression models on the full 303 pairs for which missing cofactors were imputed based on the other cofactors. Each violin plot is the result of 100 independent imputations, smoothed using a normal kernel, and its width represents the likelihood of a certain variability. Medians are shown (lines). 
a $\mathrm{BG} 505 \mathrm{~T} 332 \mathrm{~N}$

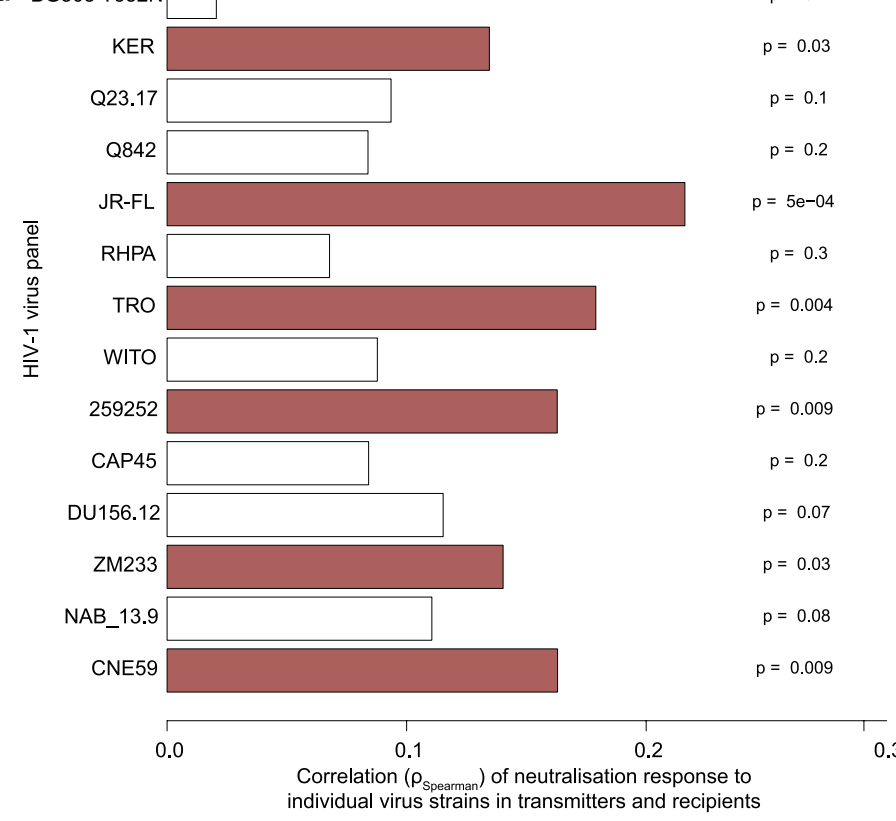

b

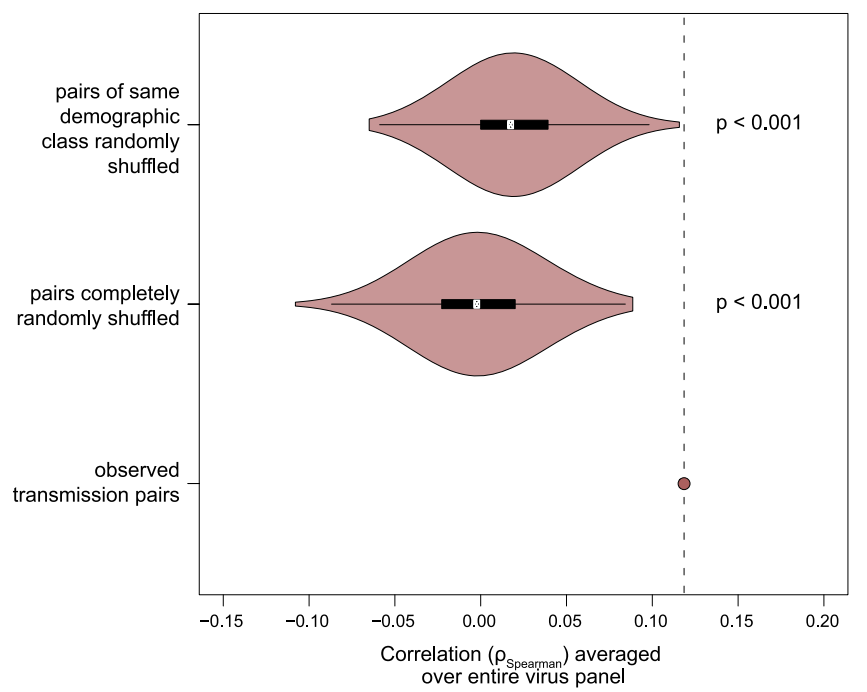

0.3 c

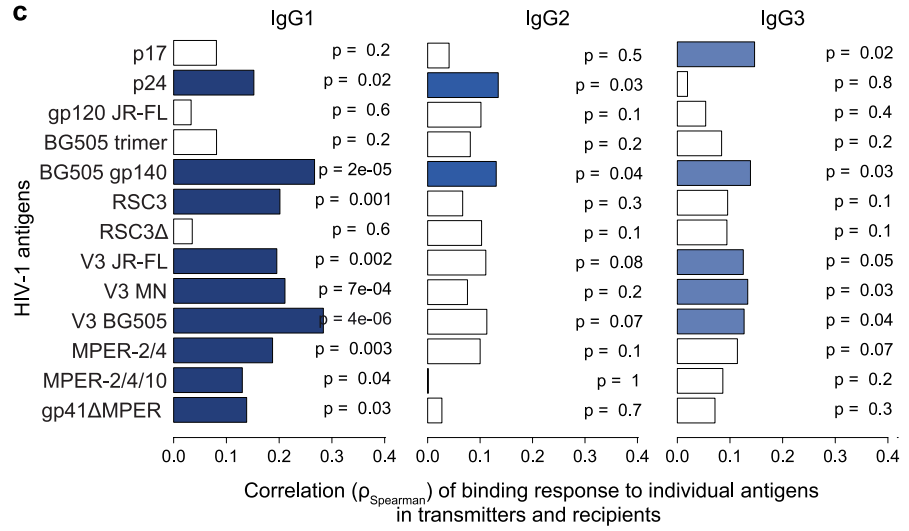

Extended Data Fig. 4 | Similarity of neutralization and antibodybinding responses in subtype-B-infected transmission pairs.

a-d, To exclude influences of the subtype of the infecting virus, as a sensitivity analysis to Fig. 1a, b, the similarity of neutralization $(\mathbf{a}, \mathbf{b})$ and antibody-binding $(\mathbf{c}, \mathbf{d})$ responses was tested for the subset of subtypeB-infected transmission pairs $(n=254)$. a, c, Spearman correlation of the neutralization-antibody-binding response to each pseudovirus or antigen. Significant correlations (two-sided $P_{\text {Spearman }}<0.05$ ) are coloured. b, d, Average Spearman correlation of antibody responses in observed transmission pairs $(n=303)$ compared to two alternative scenarios:

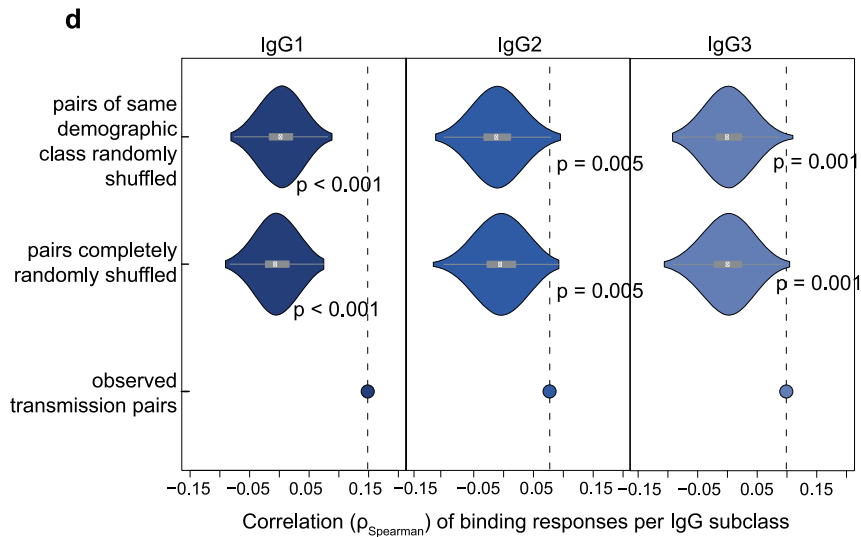

(1) completely random reassignment of recipients to transmitters and (2) random reassignment of recipients to transmitters with same demographics (subtype, ethnicity and untreated infection length). Onesided $P$ values were derived from comparison with 1,000 reassignments. Each violin plot is smoothed using a normal kernel, and its width represents the likelihood of a certain average correlation in the respective alternative scenario. The medians are shown (white dots), each box spans the IQR and each whisker extends to the most extreme value no more than $1.5 \times$ IQR from the box. 
a

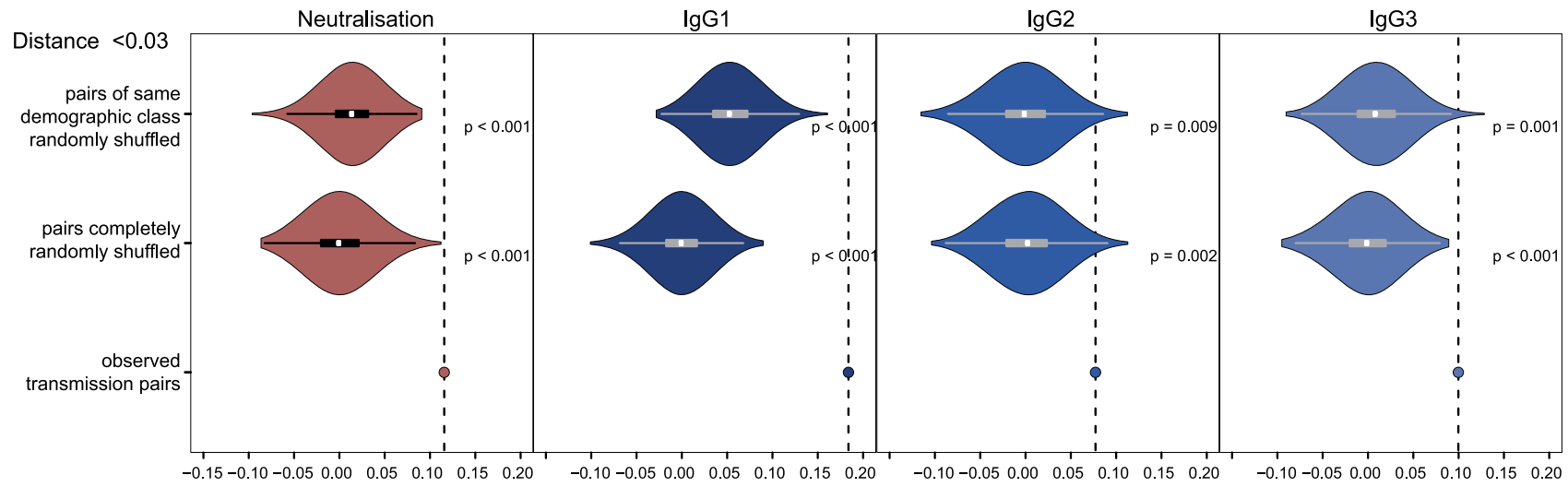

b

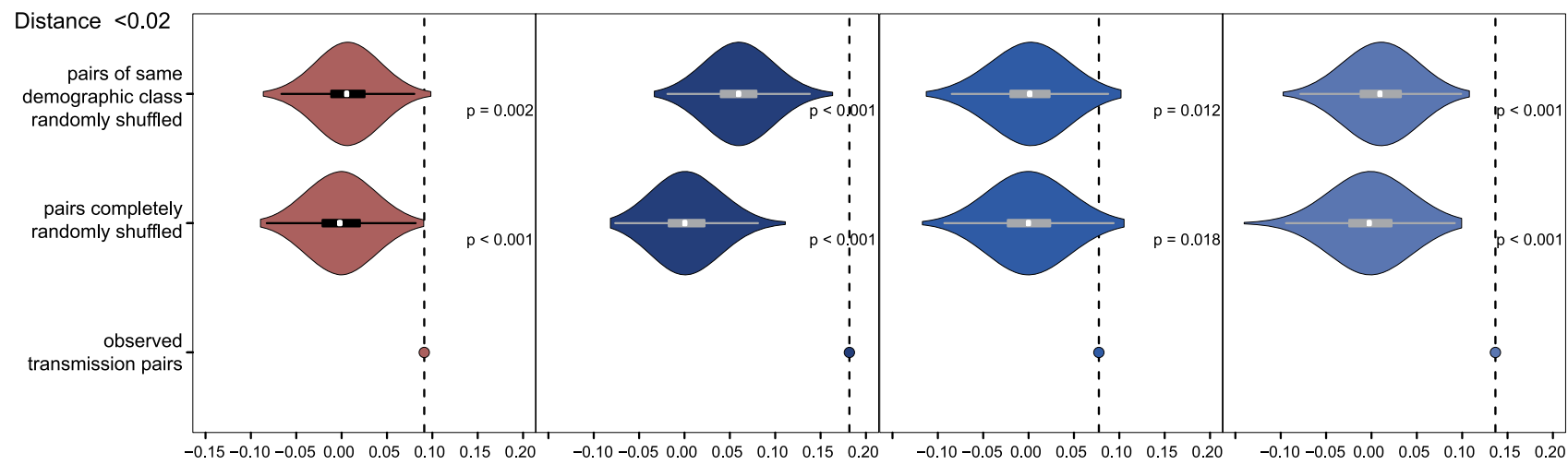

c

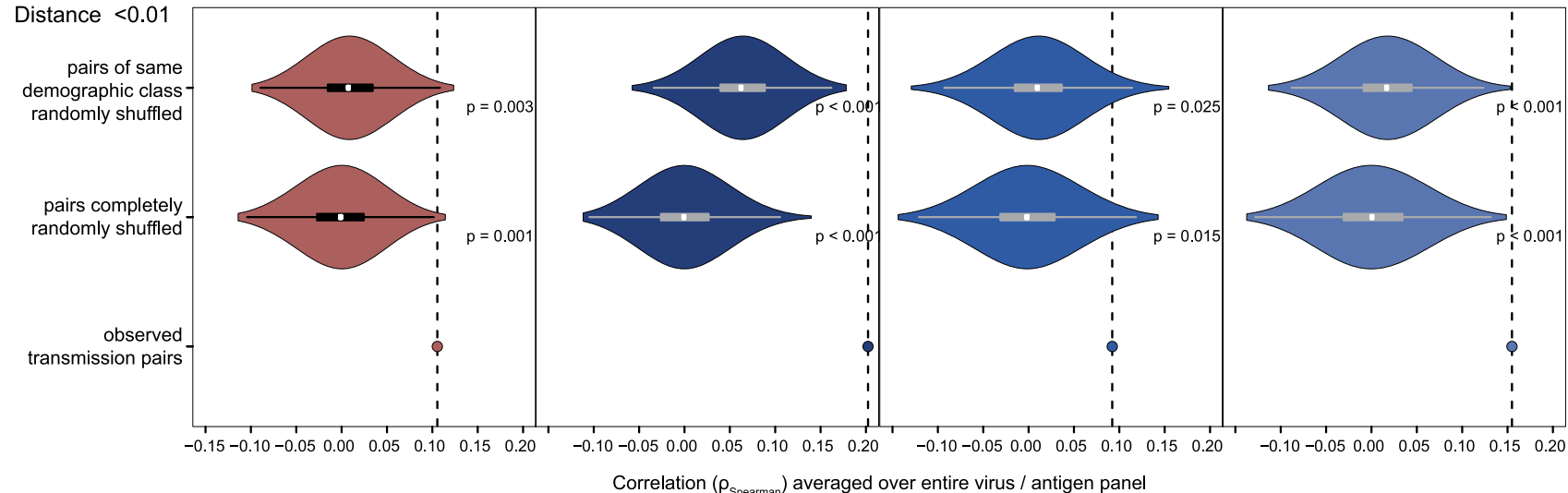

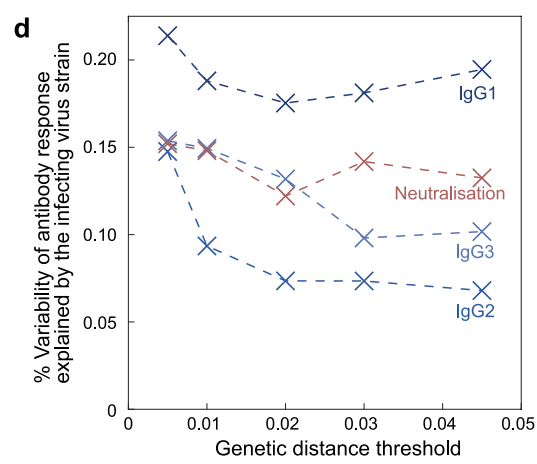

Extended Data Fig. $5 \mid$ Within-pair similarity of neutralization and binding responses remains significant for transmission pairs with lower genetic distance. a-c, As a sensitivity analysis for Fig. 1b, the average similarity of neutralization and antibody-binding responses was determined for those transmission pairs with a genetic distance $<0.03$ (a) $n=280),<0.02(\mathbf{b}, n=243)$ or $<0.01(\mathbf{c}, n=148)$, and compared to two alternative scenarios: (1) completely random reassignment of recipients to transmitters and (2) random reassignment of recipients to transmitters with same demographics (subtype, ethnicity and untreated infection length). One-sided $P$ values were derived from comparison with

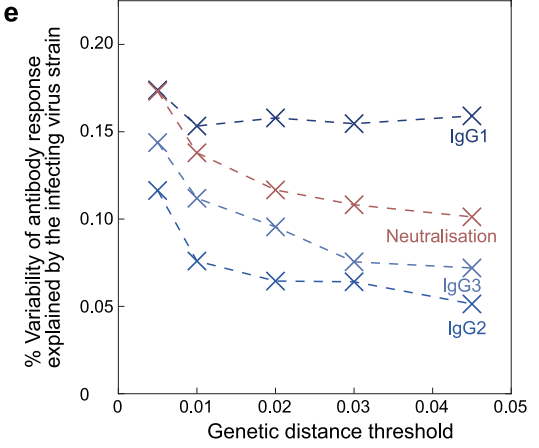

1,000 reassignments. Each violin plot is smoothed using a normal kernel, and its width represents the likelihood of a certain average correlation in the respective alternative scenario. The medians are shown (white dots), each box spans the IQR and each whisker extends to the most extreme value no more than $1.5 \times$ IQR from the box. $\mathrm{d}, \mathrm{e}, \mathrm{As}$ a sensitivity analysis for Fig. 1c, the proportion of variability in responses explained by the infecting virus, determined using unadjusted (d) and fully adjusted (e) (adjusted for duration of infection, subtype, ethnicity, log viral load and diversity) mixed-effect Tobit regression models is shown when restricting the analysis to closely related pairs (threshold on the $x$ axis). 
a

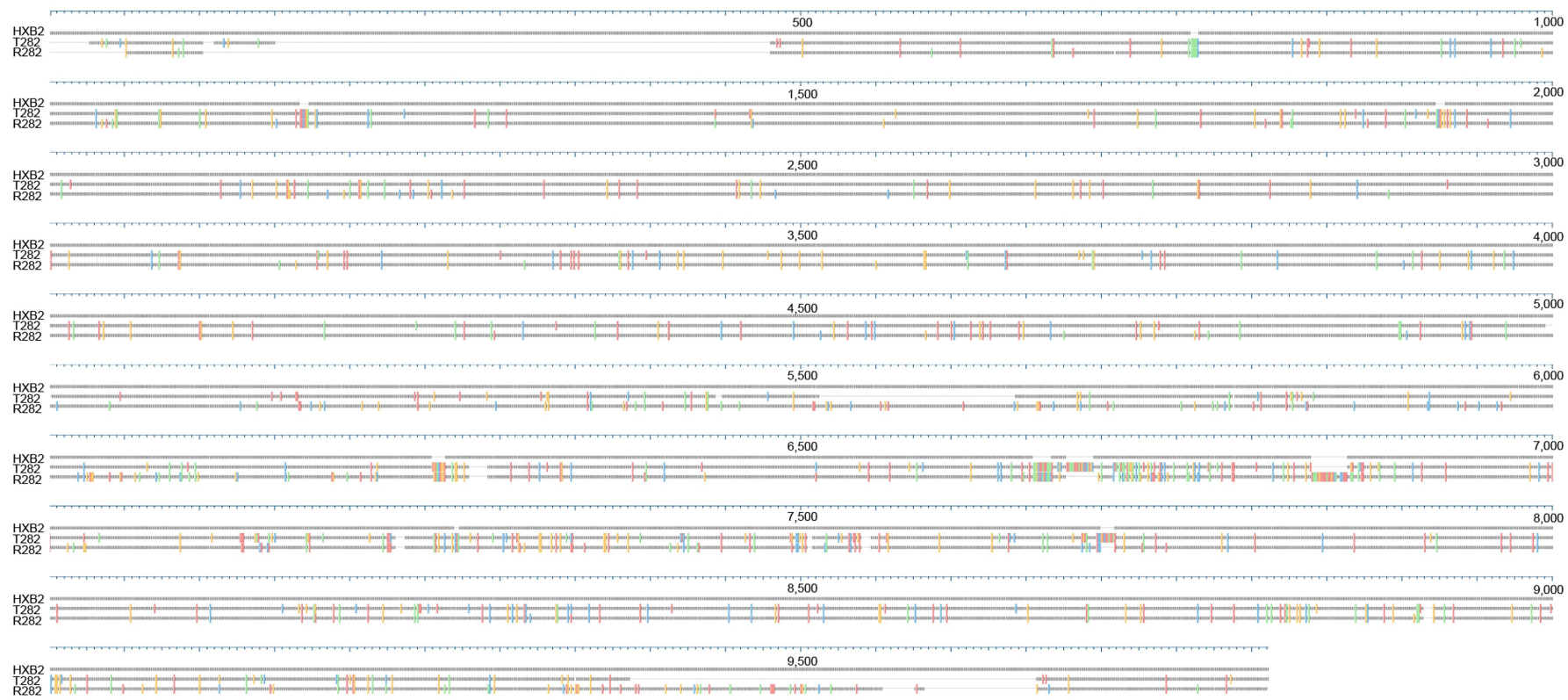

b Overview transmission pair Env sequences

\begin{tabular}{|c|c|c|c|c|c|}
\hline \multirow[t]{2}{*}{ Patient code } & \multicolumn{2}{|c|}{ Sample time point (years) } & \multirow[t]{2}{*}{ Env code } & \multirow[t]{2}{*}{ Type of data } & \multirow{2}{*}{$\begin{array}{c}\text { Genbank } \\
\text { accession number }\end{array}$} \\
\hline & $\begin{array}{l}\text { post estimated } \\
\text { infection }\end{array}$ & $\begin{array}{l}\text { post estimated } \\
\text { transmission }\end{array}$ & & & \\
\hline \multirow{3}{*}{ T282 } & \multirow{3}{*}{3.8} & \multirow{3}{*}{1.9} & T282_3.8_SGA_cl5 & Env clone SGA & MH714326 \\
\hline & & & T282_3.8_cl82 & Env clone bulk PCR & $\mathrm{MH} 714327$ \\
\hline & & & T282_3.8_con & Env consensus NGS & MH714353 \\
\hline \multirow{3}{*}{ R282 } & \multirow{3}{*}{3.4} & \multirow{3}{*}{3.4} & R282_3.4_c156 & Env clone bulk PCR & MH714324 \\
\hline & & & R282_3.4_cl61 & Env clone bulk PCR & MH714325 \\
\hline & & & R282_3.4_con & Env consensus NGS & MH714354 \\
\hline \multirow{8}{*}{ T11 } & \multirow{8}{*}{10.6} & \multirow{8}{*}{7.9} & T11_10.6_c117 & Env clone bulk PCR & MH714343 \\
\hline & & & T11_10.6_cl18 & Env clone bulk PCR & MH714344 \\
\hline & & & T11_10.6_cl19 & Env clone bulk PCR & MH714345 \\
\hline & & & T11_10.6_cl20 & Env clone bulk PCR & MH714346 \\
\hline & & & T11_10.6_c153 & Env clone bulk PCR & MH714347 \\
\hline & & & T11_10.6_cl54 & Env clone bulk PCR & MH714348 \\
\hline & & & T11_10.6_c155 & Env clone bulk PCR & MH714349 \\
\hline & & & T11_10.6_cl56 & Env clone bulk PCR & MH714350 \\
\hline \multirow[b]{2}{*}{ R11 } & \multirow[b]{2}{*}{8.1} & \multirow[b]{2}{*}{8.1} & R11_8.1_cl57 & Env clone bulk PCR & MH714351 \\
\hline & & & R11_8.1_cl58 & Env clone bulk PCR & MH714352 \\
\hline \multirow{7}{*}{ T294 } & \multirow{7}{*}{3.8} & \multirow{8}{*}{3.1} & T294_3.8_c126 & Env clone bulk PCR & MH714328 \\
\hline & & & T294_3.8_cl28 & Env clone bulk PCR & MH714329 \\
\hline & & & T294_3.8_cl30 & Env clone bulk PCR & MH714330 \\
\hline & & & T294_3.8_c173 & Env clone bulk PCR & MH714331 \\
\hline & & & T294_3.8_cl74 & Env clone bulk PCR & MH714332 \\
\hline & & & T294_3.8_c175 & Env clone bulk PCR & MH714333 \\
\hline & & & T294_3.8_c176 & Env clone bulk PCR & MH714334 \\
\hline \multirow{8}{*}{ R294 } & \multirow{8}{*}{3.8} & & R294_3.8_cl33 & Env clone bulk PCR & MH714335 \\
\hline & & \multirow{7}{*}{3.8} & R294_3.8_cl34 & Env clone bulk PCR & MH714336 \\
\hline & & & R294_3.8_cl35 & Env clone bulk PCR & MH714337 \\
\hline & & & R294_3.8_cl36 & Env clone bulk PCR & MH714338 \\
\hline & & & R294_3.8_cl89 & Env clone bulk PCR & MH714339 \\
\hline & & & R294_3.8_c190 & Env clone bulk PCR & $\mathrm{MH} 714340$ \\
\hline & & & R294_3.8_cl91 & Env clone bulk PCR & MH714341 \\
\hline & & & R294_3.8_cl92 & Env clone bulk PCR & MH714342 \\
\hline
\end{tabular}

Extended Data Fig. 6 | Full-genome comparison of T282 and R282 consensus sequences. a, Multiple sequence alignment of the full genome consensus sequences of T282 and R282 with HXB2. Nucleotide variations from HXB2 are depicted by colour: A (red), T (green), C (blue),
G (yellow); deletions by a horizontal bar. $\mathbf{b}$, Overview and accession codes of env sequences derived from three bNAb-imprinting transmission pairs depicted in Fig. 2c. Indicated transmission time estimate is based on estimated time of infection of recipient. 


\begin{tabular}{|c|c|c|c|c|c|c|c|c|c|c|}
\hline \multirow[b]{3}{*}{ bnAb epitope } & \multirow[b]{3}{*}{ Mutation } & \multirow[b]{3}{*}{ Virus } & \multicolumn{2}{|c|}{$\begin{array}{c}\text { Ratio plasma NT50 } \\
\text { wildtype/mutant }\end{array}$} & \multicolumn{6}{|c|}{$\begin{array}{c}\text { Ratio IC50 } \\
\text { mutant/wildtype }\end{array}$} \\
\hline & & & \multirow{2}{*}{$\begin{array}{c}\text { Transmitter } \\
\text { T282 }\end{array}$} & \multirow{2}{*}{$\begin{array}{c}\text { Recipient } \\
\text { R282 }\end{array}$} & \multicolumn{4}{|c|}{ CD4bs bnAbs } & \multicolumn{2}{|c|}{ V3 glycan bnAbs } \\
\hline & & & & & PGV04 & VRC01 & $\mathrm{N} \mid \mathrm{H} 45-46$ & $3 B$ BNC117 & PGT121 & PGT128 \\
\hline \multirow{3}{*}{ CD4bs specific } & \multirow{3}{*}{ N279K } & BAL_26 & 1.4 & 12.0 & 379 & 2,091 & 5,570 & 50,000 & 0.2 & 0.4 \\
\hline & & RHPA & 3.1 & 4.3 & 1,966 & 1,008 & 4,232 & 1,897 & 0.2 & 0.4 \\
\hline & & JRFL & 3.4 & 11.1 & 323 & 324 & 4,737 & 13,868 & 0.2 & 0.5 \\
\hline \multirow{4}{*}{ V3 glycan specific } & \multirow{4}{*}{$\begin{array}{c}\text { D325A } \\
\& \\
\text { N332A }\end{array}$} & HIV-001428-2 & 2.3 & 0.3 & 0.9 & 4.2 & 2.3 & 0.4 & 905 & 1,178 \\
\hline & & RHPA & 2.7 & 0.5 & 0.4 & 1.1 & 0.6 & 0.1 & 4,616 & 2,404 \\
\hline & & JRCSF & 3.6 & 0.1 & 2.6 & 1.3 & 1.9 & 0.1 & 996 & 12,071 \\
\hline & & JRFL & 1.1 & 0.5 & 0.5 & 0.5 & 1.2 & 0.1 & 1,145 & 846 \\
\hline
\end{tabular}

$>2$ fold decrease in NT50 CD4bs mutant $/>10$ fold increase in IC50 CD4bs mutant

$>2$ fold decrease in NT50 $0^{\mathrm{V} 3}$ mutant $/>10$ fold increase in $1 \mathrm{C} 50^{\mathrm{V} / 3}$ mutant

\begin{tabular}{|l|cc|}
\hline Antibody response similarity threshold & 0.4 & 0.5 \\
\hline Neutralisation strength threshold & 4 & 5 \\
\hline Variables & Odds Ratio $(95 \%$ Confidence Interval $)$ \\
\hline Mean log viral load & \multicolumn{2}{|c|}{$p$-Value } \\
& $1.2(0.51-2.8)$ & $1.2(0.31-5)$ \\
Difference in log viral load & $p=0.68$ & $p=0.75$ \\
& $0.92(0.4-2.1)$ & $1.1(0.33-3.8)$ \\
Genetic distance & $p=0.85$ & $p=0.87$ \\
& $2(1.2-3.1)$ & $1.7(0.82-3.5)$ \\
& $p=0.004$ & $p=0.15$ \\
\hline
\end{tabular}

c
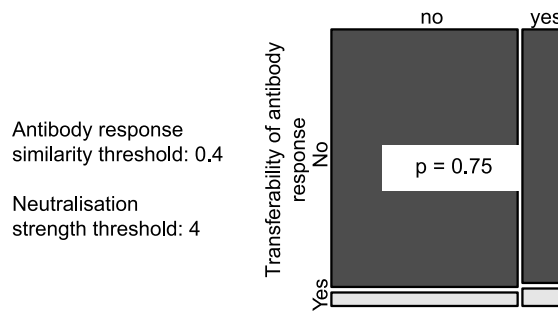

Acute transmission

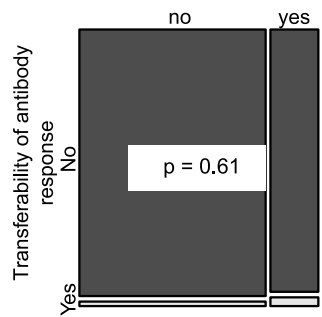

Acute transmission

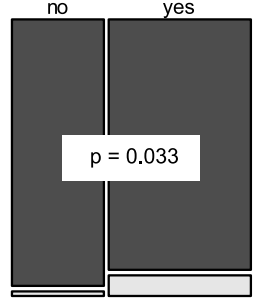

$>3$ years of virus evolution

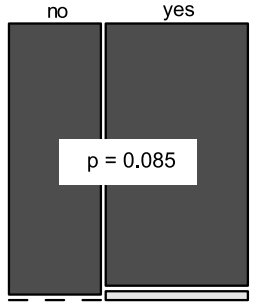

$>3$ years of virus evolution

Extended Data Fig. 7 Mutant virus neutralization mapping and distribution of transmission pair characteristics among pairs with and without transferability of antibody response. a, Changes in neutralization activity of wild-type and mutant viruses were compared for six bNAbs (four targeting the CD4 binding site, two targeting the V3 glycan) and for the plasma of the elite-neutralizing pair. Increases in mutant half-maximum inhibitory concentration $\left(\mathrm{IC}_{50}\right)$ values $>$ tenfold and decreases in mutant half-maximum neutralization titre $\left(\mathrm{NT}_{50}\right)$ values $>$ two fold appear coloured. $\mathbf{b}, \mathbf{c}$, Association of transmission pair characteristics and transferability of antibody response in $n=303$ transmission pairs. Transferability of antibody responses was determined

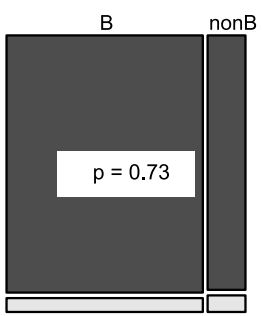

Subtype

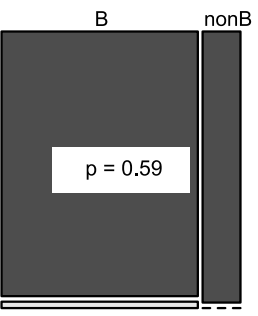

Subtype

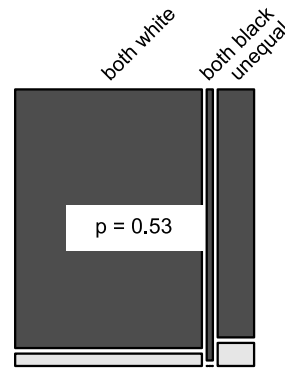

Ethnicity

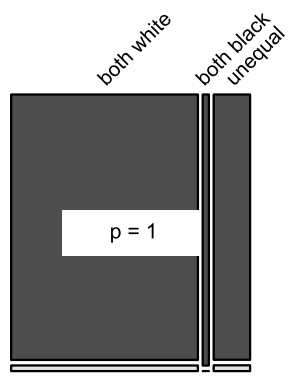

Ethnicity

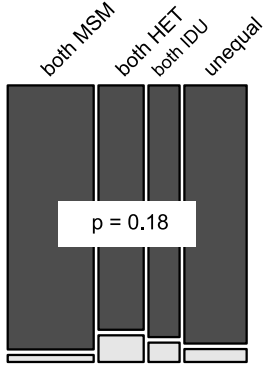

Transmission risk group

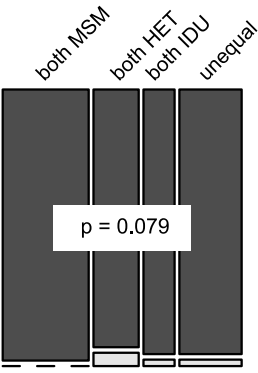

Transmission risk group according to relatively liberal (strength threshold $=4$ and similarity threshold $=0.4$ ) and strict criteria (strength threshold $=5$ and similarity threshold $=0.5)$. $\mathbf{b}$. Influence of continuous variables (mean $\log _{10}$ HIV-1 RNA in the transmission pair, the difference of these $\log _{10}$ RNA values, and the genetic distance in the pair) tested by univariable logistic regression. c, Influence of categorical variables (acute infection, long virus evolution, infecting subtype, ethnicity and transmission mode) tested by two-tailed Fisher's exact test. The area of a rectangle corresponds to the number of pairs with the respective characteristic. MSM, men who have sex with men; HET, heterosexual transmission; IDU, intravenous drug users. 


\section{RESEARCH LETTER}

Step 1: Establish antibody response similarity index

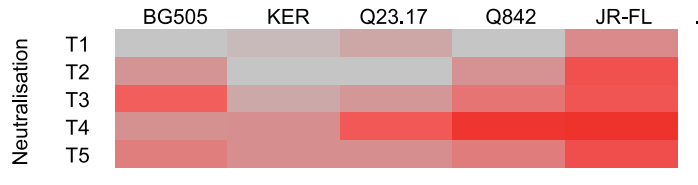

$\ldots$
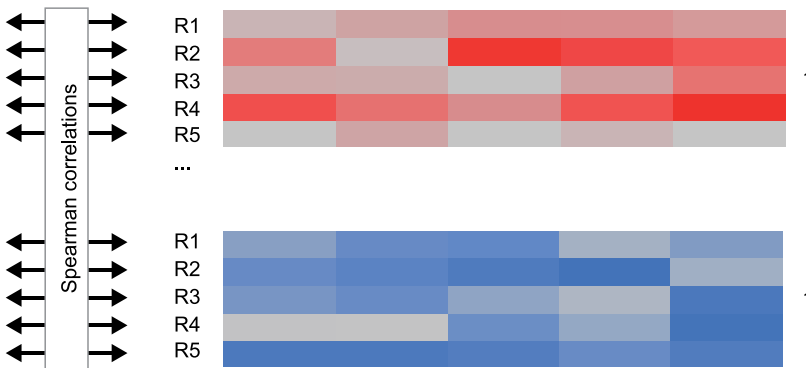

14 parameters

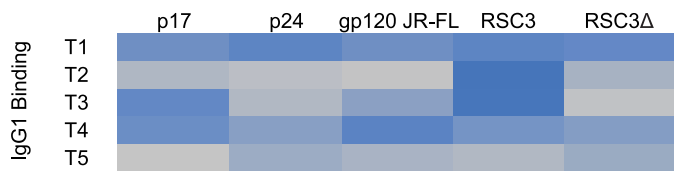

Similarity Index:

- Compare within pair similarity of neutralisation activity and IgG1 responses

- Calculate combined Spearman rho (Neutralisation and Binding)

\section{Step 2: Establish neutralisation strength of pair}

- calculate lowest neutralisation score of pair

Step 3: Determine pairs with high similarity of antibody response and high neutralisation score

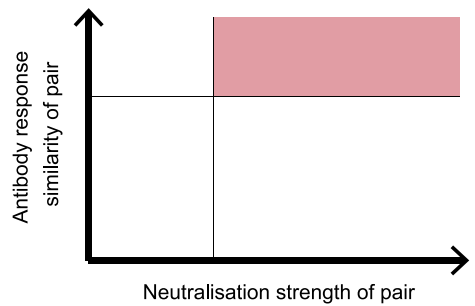

Step 4: Probe thresholds of similarity index and high neutralisation score to define HIV-1 strains with higher than random capacity to imprint neutralising antibody responses

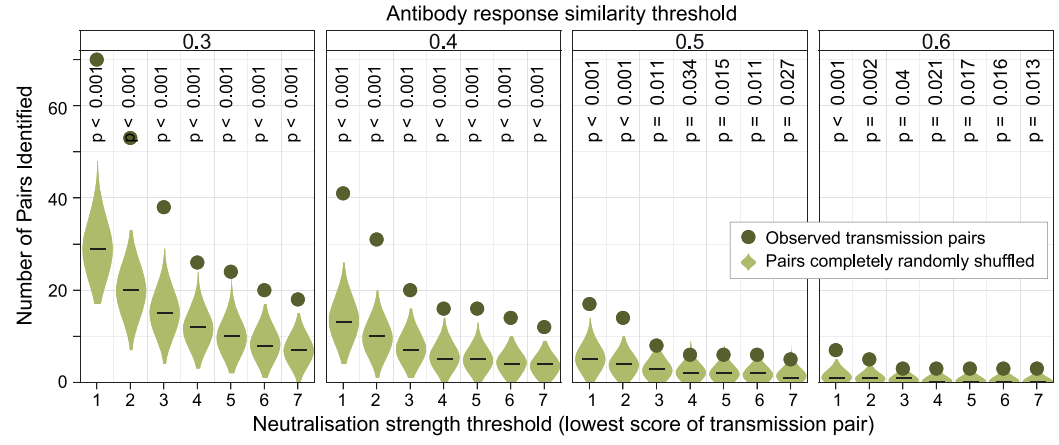

Extended Data Fig. 8 | Systematic strategy to trace HIV-1 strains with bNAb-imprinting capacity. HIV-1 strains that have the capacity to induce bNAb activity can be identified by measuring the similarity and the strength of the antibody response in each pair. A stepwise description of the approach is shown. See Methods 'Systematic strategy to trace HIV-1 strains with bNAb-imprinting capacity' for details. 
Extended Data Table 1 | Patient demographics of the study population and entire population in the Swiss 4.5K screen

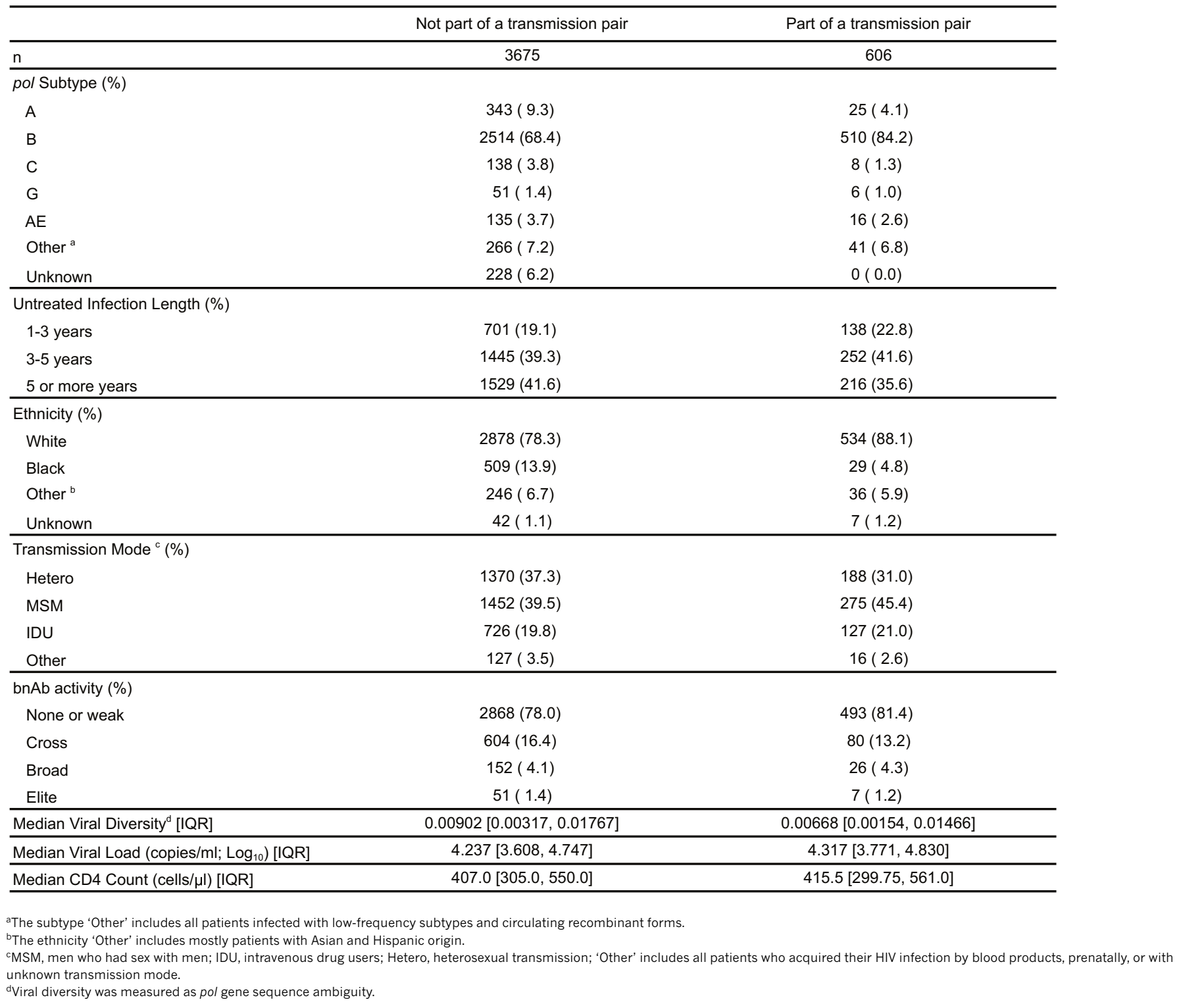


Extended Data Table 2 | Proportion of variability of neutralization and binding responses explained by the infecting virus strain using variously adjusted mixed-effect Tobit models

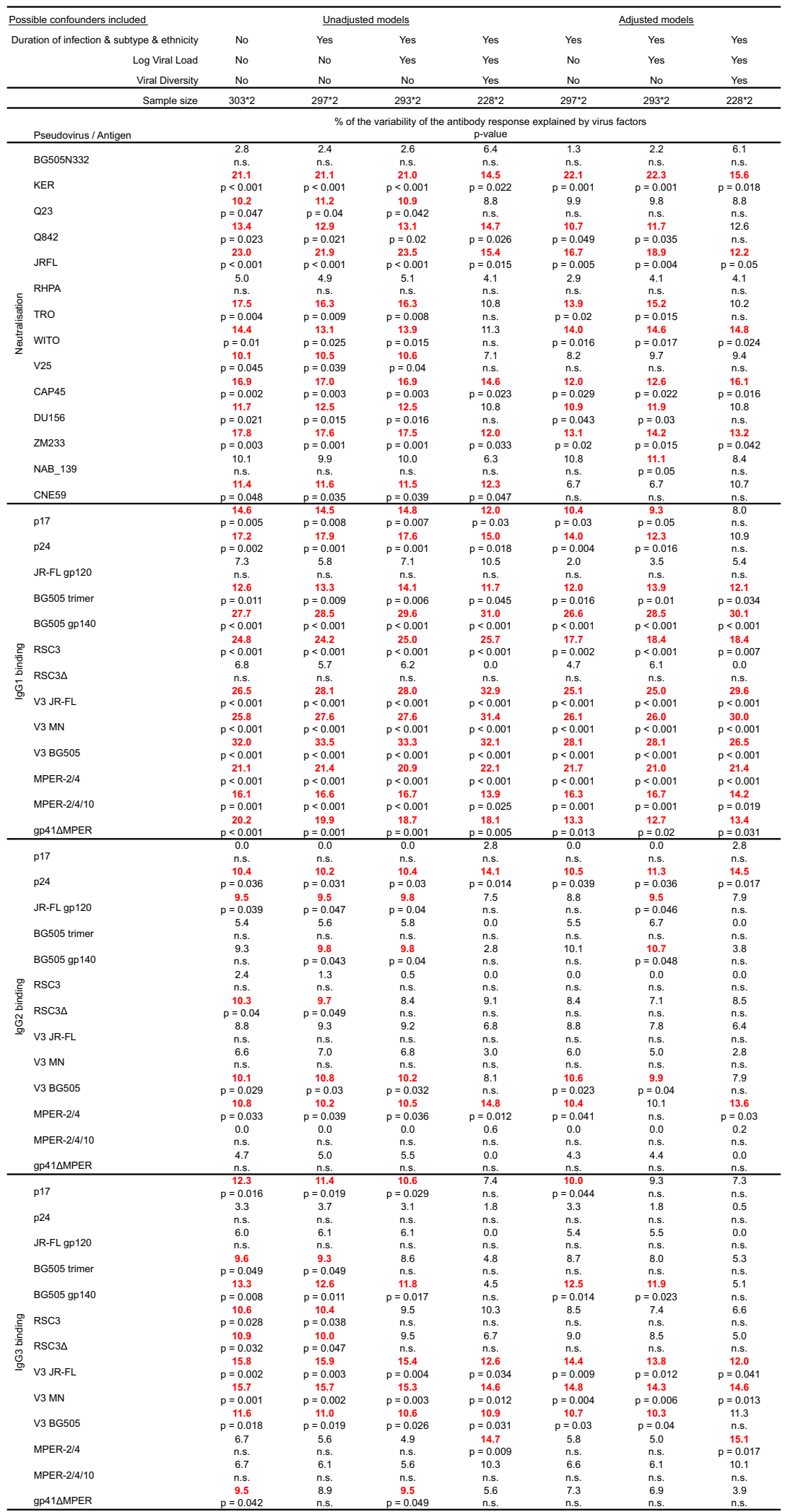

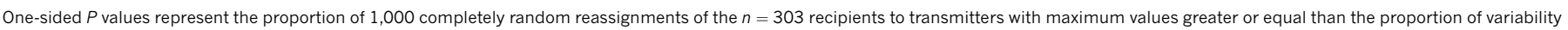
observed for the transmission pair cohort. Significant results $(P<0.05)$ are shown in red. 


\section{Reporting Summary}

Nature Research wishes to improve the reproducibility of the work that we publish. This form provides structure for consistency and transparency in reporting. For further information on Nature Research policies, see Authors \& Referees and the Editorial Policy Checklist.

\section{Statistical parameters}

When statistical analyses are reported, confirm that the following items are present in the relevant location (e.g. figure legend, table legend, main text, or Methods section).

n/a Confirmed

$\square$ The exact sample size $(n)$ for each experimental group/condition, given as a discrete number and unit of measurement

$\square$ An indication of whether measurements were taken from distinct samples or whether the same sample was measured repeatedly

$\searrow$ The statistical test(s) used AND whether they are one- or two-sided

Only common tests should be described solely by name; describe more complex techniques in the Methods section.

$\square$ A description of all covariates tested

$\square$ A description of any assumptions or corrections, such as tests of normality and adjustment for multiple comparisons

$\square$ A full description of the statistics including central tendency (e.g. means) or other basic estimates (e.g. regression coefficient) AND

variation (e.g. standard deviation) or associated estimates of uncertainty (e.g. confidence intervals)

$\varnothing$ For null hypothesis testing, the test statistic (e.g. $F, t, r$ ) with confidence intervals, effect sizes, degrees of freedom and $P$ value noted

Give $P$ values as exact values whenever suitable.

Х $\square$ For Bayesian analysis, information on the choice of priors and Markov chain Monte Carlo settings

Х $\square$ For hierarchical and complex designs, identification of the appropriate level for tests and full reporting of outcomes

\ $\square$ Estimates of effect sizes (e.g. Cohen's $d$, Pearson's $r$ ), indicating how they were calculated

$\varnothing$ Clearly defined error bars

State explicitly what error bars represent (e.g. SD, SE, CI)

Our web collection on statistics for biologists may be useful.

\section{Software and code}

Policy information about availability of computer code

Data collection BlastN

Data analysis R, Python 2.7, Stata15, FastTree, APE version 3.1, freebayes version 1.1.0, smalt version 0.7.6, lofreq version 2.1.3a, RAXML 8.2.11

For manuscripts utilizing custom algorithms or software that are central to the research but not yet described in published literature, software must be made available to editors/reviewers upon request. We strongly encourage code deposition in a community repository (e.g. GitHub). See the Nature Research guidelines for submitting code \& software for further information.

\section{Data}

Policy information about availability of data

All manuscripts must include a data availability statement. This statement should provide the following information, where applicable:

- Accession codes, unique identifiers, or web links for publicly available datasets

- A list of figures that have associated raw data

- A description of any restrictions on data availability

The antibody response and patient data reported in this paper are completely tabulated in Supplementary Datasets 1-2. Sequence data of Env consensus and Env clones reported in Fig. $2 \mathrm{c}$ are deposited in GenBank. Accession codes are listed in Extended Data Fig. 6b. The raw sequencing files of the Illumina full HIV sequencing 


\section{Field-specific reporting}

Please select the best fit for your research. If you are not sure, read the appropriate sections before making your selection.

\Life sciences $\quad \square$ Behavioural \& social sciences $\quad \square$ Ecological, evolutionary \& environmental sciences

For a reference copy of the document with all sections, see nature.com/authors/policies/ReportingSummary-flat.pdf

\section{Life sciences study design}

All studies must disclose on these points even when the disclosure is negative.

Sample size

No sample-size calclutaion was performed. We included all individuals from the initial Swiss 4.5k screen (Rusert, Kouyos, Nat Med 2016), for which also binding antibody responses were available (collected in Kadelka et al., J Exp Med 2018). This was the case for 4,281 individuals. These 4,281 individuals were used to phylogenetically identify transmission pairs, and this led to the identification of a cohort of 303 putative transmission pairs ( the largest thus far described transmission cohort) , which constitutes the basis of this analysis.

Data exclusions All 303 identified transmission pairs are used throughout the study. No data was excluded.

Replication Neutralization assays were performed in 2-3 independent, succesful assay replicates. The same source materila ( plasma sample) was used in all replicates. Data from all replicates are included in the analysis.

Collection of binding data and reproducibility of the binding assay are described in Kadelka et al., J Exp Med 2018 (DOI: 10.1084/ jem.20180246) and Liechti et al., JIM 2017 (DOI: 10.1016/j.jim.2017.12.003). Due to the high-throughput analysis binding data are based on single measurements as described there

Randomization Personnel who conducted the neutralization assays of the transmitter cohort had no information on the transmitter/recipient status at the time of testing. There was thus no randomization/allocation of the 606 plasma samples into specific groups performed. Sample testing followed purely practical considerations according to freezer location of the samples (all samples were stored at $-80^{\circ} \mathrm{C}$ from the previous analysis in the Swiss $4.5 \mathrm{~K}$ Study in boxes containing up to 96 samples).

Blinding $\quad$ Personnel who conducted the neutralization assay and the binding assay had no information on allocation to the transmitter and recipient groups at the time of analysis. Investigators were also blinded regarding patient information from the Swiss HIV Cohort and Zurich Primary HIV Cohort Databases: patients are annonymized in the SHCS and ZPHI databases.

\section{Reporting for specific materials, systems and methods}

Materials \& experimental systems

\begin{tabular}{l|l}
\hline $\mathrm{n} / \mathrm{a}$ & Involved in the study \\
$\square$ Unique biological materials
\end{tabular}

\begin{tabular}{l|l}
\multicolumn{2}{l}{ Methods } \\
\hline n/a & Involved in the study \\
Х & $\square$ ChIP-seq \\
$\searrow$ & $\square$ Flow cytometry \\
$\bigotimes$ & $\square$ MRI-based neuroimaging
\end{tabular}

\section{Antibodies}

bnAbs used in this study were ( clone name/ and source reference): PG9 (Walker 2009 Science/19729618), PG16 (Walker 2009 Science/19729618), PGDM1400 (Sok PNAS 2014/25422458), PGT145 (Walker 2011 Nature/21849977), CAP256-09 (Doria-Rose 2014 Nature/24590074), CAP256-25 (Doria-Rose 2016 J Virol/26468542), PGT121 (Walker 2011 Nature/21849977), PGT128 (Walker 2011 Nature/21849977), PGT130 (Walker 2011 Nature/21849977), PGT135 (Walker 2011 Nature/21849977), 10-1074 (Mouquet 2012 PNAS/23115339), 2 G12 (Trkola 1996 J Virol/8551569), VRC01 (Wu 2010 Science/20616233),PGV04 (Wu 2011 Science/21835983), NIH45-46 (Scheid 2011 Science/21764753), 3BNC117(Scheid 2011 Science/21764753), VRC_CH31 (Wu 2011 Science/21835983), HJ16 (Corti 2010 PloS ONE/20098712), b12 (Barbas III 1992 PNAS/1384050), PGT151 (Falkowska 2014 Immunity/24768347), 35022 (Huang 2014 Nature/25186731), 8ANC195 (Scheid 2011 Science/21764753),2F5 (Muster 1993 J 
Validation

Virol/7514684); 4E10 (Stiegler 2001 AIDS Res Hum Retroviruses/11788027); $10 E 8$ (Huang 2012 Nature/23151583).Source references are also listed in Supplementary Dataset 2. We thank D. Burton, J. Mascola, M. Nussenzweig, M. Connors, D. Katinger, P. Moore and L. Morris for providing antibodies and/or antibody expression plasmids for this study either directly or via the NIH AIDS Research and Reference Reagent Program (NIH ARP).

Antibodies were prepared from expression plasmids encoding the published antibody sequences as described in Tiller 2008 (ref 31). As the antibody preparations used in the current study were from different sources we validated their performance against the full virus panel shown in Supplementary Dataset 2 and Fig. 2e. Observed activities of all Ab preparations matched the reported activity of the respective bnAbs against a range of HIV-1 strains (see CATNAP https://www.hiv.lanl.gov/components/ sequence/HIV/neutralization/main.comp), and were thus rated as valid.

\section{Eukaryotic cell lines}

Policy information about cell lines

Cell line source(s)

293-T cells (American Type Culture Collection (ATCC)). TZM-bl cells (NIH AIDS Reagent Program)

Authentication

We did not perform a specific authentication. Both cell lines are routinely used in our laboratory for years. Cell cultures are periodically (every 3-4 months) restarted from freezer stocks.

Mycoplasma contamination

All used cell stocks tested negative for mycoplasma.

Commonly misidentified lines

(See ICLAC register)

no commonly misidentified cell lines were used

\section{Animals and other organisms}

Policy information about studies involving animals; ARRIVE guidelines recommended for reporting animal research

Laboratory animals

Wild animals

Field-collected samples none used

none used

none used

\section{Human research participants}

\section{Policy information about studies involving human research participants}

Population characteristics

As detailed in the methods section, specimen and clinical data from two pre-existing longituidinal, natural history HIV cohorts, the SHCS and the ZPHI study, were used. All patients were HIV-1 infected adults. A prerequisite for inclusion in the current study was that patients were part of the starting cohort described in Rusert Nat Med 2016 and Kadelka JEM 2018. Amongst the 4,281 individuals described in Kadelka JEM 2018 the 303 transmission pairs were identified. Patient data analyzed included: gender, ethnicity, HIV-1 subtype, length of infection (including estimated date of infection), CD4 levels, viral load, neutralisation activity, HIV-binding antibody activity), pol gene sequence and diversity

Recruitment

There was no recruitment for our study. We used biobanked samples and data collected from two pre-existing longituidinal cohorts, the SHCS and the ZPHI study as detailed in the methods section. Patient selection was given through the starting cohort described in Rusert Nat Med 2016 and Kadelka JEM 2018 